\title{
Equivalence between entropy and renormalized solutions for parabolic equations with smooth measure data
}

\author{
Jérôme DRONIOU \\ Département de Mathématiques CC 051 \\ Université Montpellier II \\ Place Eugène Bataillon \\ 34095 Montpellier cedex 5 \\ France \\ email: droniou@math.univ-montp2.fr \\ Alain PRIGNET \\ Mathématiques \\ Université de Marne la Vallée \\ 5, boulevard Descartes Cité Descartes - Champs-sur-Marne \\ 77454 Marne-la-Vallée cedex 2 \\ France \\ email: alain.prignet@univ-mlv.fr
}

\begin{abstract}
We consider the nonlinear heat equation (with Leray-Lions operators) on an open bounded subset of $\mathbf{R}^{N}$ with Dirichlet homogeneous boundary conditions. The initial condition is in $L^{1}$ and the right hand side is a smooth measure. We extend a previous notion of entropy solutions and prove that they coincide with the renormalized solutions.
\end{abstract}

2000 Mathematics Subject Classification: 35K55, 35D99.

Key words: parabolic equations, measure, capacity, renormalized solutions, entropy solutions.

\section{Introduction}

We consider the parabolic equation

$$
\begin{cases}u_{t}-\operatorname{div}(a(t, x, u, \nabla u))=\mu & \text { in } Q=] 0, T[\times \Omega, \\ u=0 & \text { on }] 0, T[\times \partial \Omega, \\ u(0)=u_{0} & \text { in } \Omega\end{cases}
$$


where $T>0, \Omega$ is a bounded open subset of $\mathbf{R}^{N}(N \geq 1),-\operatorname{div}(a(t, x, u, \nabla u))$ is a Leray-Lions operator, $u_{0} \in L^{1}(\Omega)$ and $\mu$ is a bounded measure on $Q$ which is absolutely continuous with respect to the parabolic capacity associated with the operator $-\operatorname{div}(a(t, x, u, \nabla u))$ (see below the precise hypotheses).

Under the general assumption that $\mu$ and $u_{0}$ are bounded measures, the existence of a distributional solution can be proved (see [4, 3]). However, due to the lack of regularity of the solutions, this formulation is not strong enough to provide uniqueness. Hence new concepts of solution have been defined for integrable right hand sides (see $[2,10,6]$ ). For a more complete presentation and references, see $[6]$.

In [6], the notion of renormalized solution has been developped in the case of bounded measures absolutely continuous with respect to the capacity. We extend here the notion of entropy solutions (defined in [10]) to these right-hand sides and we prove that these solutions are the same as the renormalized solutions.

Notice that we also slightly extend the notion of renormalized solution to take into account that, on the contrary to [6], our Leray-Lions operator also depends on $u$. In particular, in the case we consider, uniqueness of the renormalized (or entropy) solution is not certain, and we therefore prove that any entropy solution is a renormalized solution (and vice-versa).

\section{Hypotheses and definitions}

We take $T>0$ and $\Omega$ a bounded open subset of $\mathbf{R}^{N}$, and we denote $Q=10, T[\times \Omega$. Let $p \in] 1, \infty\left[\right.$; we take $\left.\left.p^{*} \in\right] p, \infty\right]$ such that $W_{0}^{1, p}(\Omega)$ is embedded in $L^{p^{*}}(\Omega)$ (i.e. $p^{*}=N p /(N-p)$ if $p<N, p^{*}<\infty$ if $p=N$ and $p^{*}=\infty$ if $\left.p>N\right)$.

The hypotheses on the operator in (1.1) are the following:

$$
a:] 0, T\left[\times \Omega \times \mathbf{R} \times \mathbf{R}^{N} \rightarrow \mathbf{R}^{N}\right. \text { is a Caratheodory function, }
$$

$$
\begin{gathered}
\exists \Lambda \in L^{1}(Q), \exists \alpha>0 \text { such that } a(t, x, s, \xi) \cdot \xi \geq \alpha|\xi|^{p}-\Lambda(t, x) \\
\text { for a.e. }(t, x) \in Q \text { and all }(s, \xi) \in \mathbf{R} \times \mathbf{R}^{N},
\end{gathered}
$$

$$
\begin{gathered}
\exists \beta>0, \exists b \in L^{p^{\prime}}(Q), \exists \nu<(p-1)\left(1-\frac{1}{p^{*}}\right) \text { such that } \\
\qquad|a(t, x, s, \xi)| \leq \beta\left(b(t, x)+|s|^{\nu}+|\xi|^{p-1}\right) \\
\text { for a.e. }(t, x) \in Q \text { and all }(s, \xi) \in \mathbf{R} \times \mathbf{R}^{N} \\
(a(t, x, s, \xi)-a(t, x, s, \eta)) \cdot(\xi-\eta)>0
\end{gathered}
$$

for a.e. $(t, x) \in Q$ and all $(s, \xi, \eta) \in \mathbf{R} \times \mathbf{R}^{N} \times \mathbf{R}^{N}$ such that $\xi \neq \eta$.

Remark 2.1 As examples of such functions, we can take $a(t, x, s, \xi)=|\xi|^{p-2} \xi$ (the $p$-Laplacian), but also $a(t, x, s, \xi)=|\xi|^{p-2} \xi+\phi(t, x, s)+\psi(t, x, s)|\xi|^{q-2} \xi$, 
where $q \in] 1, p\left[, \phi\right.$ and $\psi$ are Caratheodory functions, $\sup _{s \in \mathbf{R}}|\phi(\cdot, \cdot, s)| \in L^{p^{\prime}}(Q)$, $\psi \geq 0$ and $|\psi(t, x, s)|^{\frac{p-1}{p-q}} \leq C\left(h(t, x)+|s|^{\nu}\right)$ with $h \in L^{p^{\prime}}(Q)$.

We denote by $\mathcal{M}_{0}(Q)$ the set of bounded measures on $Q$ which are absolutely continuous with respect to the $p$-parabolic capacity. We will not detail the results concerning this capacity and $\mathcal{M}_{0}(Q)$ (see [6]), we only recall the following.

Let us define $V=W_{0}^{1, p}(\Omega) \cap L^{2}(\Omega)$, and $W=\left\{u \in L^{p}(0, T ; V) \mid u_{t} \in\right.$ $\left.L^{p^{\prime}}\left(0, T ; V^{\prime}\right)\right\}$, endowed with the norm $\|u\|_{W}=\|u\|_{L^{p}(0, T ; V)}+\left\|u_{t}\right\|_{L^{p^{\prime}}\left(0, T ; V^{\prime}\right)}$. If $U \subset Q$ is an open set, we define the parabolic capacity of $U$ as $\operatorname{cap}_{p}(U)=$ $\inf \left\{\|u\|_{W}: u \in W, u \geq \chi_{U}\right.$ almost everywhere in $\left.Q\right\}$ (we use the convention $\inf \emptyset=+\infty$ ); then for any borelian subset $B \subset Q$ the definition is extended by setting: $\operatorname{cap}_{p}(B)=\inf \left\{\operatorname{cap}_{p}(U), U\right.$ open subset of $\left.Q, B \subset U\right\} . \mathcal{M}_{0}(Q)$ is defined as the set of bounded measures $\mu$ satisfying $\mu(E)=0$ for every subset $E \subset Q$ such that $\operatorname{cap}_{p}(E)=0$. The next theorem states the main property of $\mathcal{M}_{0}(Q)$.

Theorem 2.2 If $\mu \in \mathcal{M}_{0}(Q)$, then there exist $f \in L^{1}(Q), G_{1} \in L^{p^{\prime}}(Q)^{N}$ and $g_{2} \in L^{p}\left(0, T ; W_{0}^{1, p}(\Omega) \cap L^{2}(\Omega)\right)$ such that

$\int_{Q} \varphi d \mu=\int_{Q} f \varphi d t d x+\int_{Q} G_{1} \cdot \nabla \varphi d t d x-\int_{Q} \varphi_{t} g_{2} d t d x, \quad \forall \varphi \in C_{c}^{\infty}([0, T] \times Q)$. $\left(f,-\operatorname{div}\left(G_{1}\right), g_{2}\right)$ is called a decomposition of $\mu$ (it is not unique).

For $k \geq 0$, we let $T_{k}(s)=\max (-k, \min (k, s))$ the truncature at levels $k$ and $-k$ and we let $\Theta_{k}(s)=\int_{0}^{s} T_{k}(\tau) d \tau$ (remark that $\Theta_{k}(s) \geq 0$ for all $s$ and all $k \geq 0$ ). Let us now state the definition of a renormalized solution for (1.1) (a slight generalization of the one in $[6]$ ).

Definition 2.3 Under hypotheses (2.1)-(2.4), if $u_{0} \in L^{1}(\Omega), \mu \in \mathcal{M}_{0}(Q)$ and $\left(f,-\operatorname{div}\left(G_{1}\right), g_{2}\right)$ is a decomposition of $\mu$ according to Theorem 2.2 , then a renormalized solution to (1.1) is a measurable function $u$ such that

$$
\begin{gathered}
u-g_{2} \in L^{\infty}\left(0, T ; L^{1}(\Omega)\right), T_{k}\left(u-g_{2}\right) \in L^{p}\left(0, T ; W_{0}^{1, p}(\Omega)\right) \text { for all } k \geq 0, \\
\lim _{n \rightarrow \infty} \int_{\left\{n \leq\left|u-g_{2}\right| \leq n+1\right\}}|\nabla u|^{p} d t d x=0
\end{gathered}
$$

and, for all $S \in W^{2, \infty}(\mathbf{R})$ such that $S^{\prime}$ has a compact support,

$$
\begin{gathered}
\left(S\left(u-g_{2}\right)\right)_{t}-\operatorname{div}\left(a(t, x, u, \nabla u) S^{\prime}\left(u-g_{2}\right)\right) \\
+S^{\prime \prime}\left(u-g_{2}\right) a(t, x, u, \nabla u) \cdot \nabla\left(u-g_{2}\right) \\
=S^{\prime}\left(u-g_{2}\right) f+S^{\prime \prime}\left(u-g_{2}\right) G_{1} \cdot \nabla\left(u-g_{2}\right) \\
-\operatorname{div}\left(S^{\prime}\left(u-g_{2}\right) G_{1}\right) \quad \text { in } \mathcal{D}^{\prime}(Q), \\
\quad S\left(u-g_{2}\right)(0)=S\left(u_{0}\right) \text { in } L^{1}(\Omega) .
\end{gathered}
$$


Remark 2.4 It is known that, if $v=u-g_{2}$ satisfies $T_{k}(v) \in L^{p}\left(0, T ; W_{0}^{1, p}(\Omega)\right)$ for all $k \geq 0$, then there exists a unique measurable vector-valued function $\nabla v$ such that $\nabla\left(T_{k}(v)\right)=\mathbf{1}_{\{|v| \leq k\}} \nabla v$ for all $k \geq 0$ (see [1] for the elliptic case); hence, if $u$ satisfies (2.5), we can define the function $\nabla\left(u-g_{2}\right)$ and, since the gradient of $g_{2}$ is a function, this also gives a gradient $\nabla u=\nabla\left(u-g_{2}\right)+\nabla g_{2}: Q \rightarrow \mathbf{R}^{N}$ for $u$.

We will see in section 4 that (2.7), with this definition of $\nabla u$, and (2.8) make sense.

Remark 2.5 It is proved in [6] (in the case where $a$ does not depend on $u$ ) that this definition of renormalized solution does not depend on the decomposition of $\mu$ : if a function is a renormalized solution for a particular decomposition of $\mu$, then it is a renormalized solution for any decomposition of $\mu$. This is still true if $a$ depends on $u$ : let $u$ be a renormalized solution for a decomposition of $\mu$ and consider, as in Section $6.2, \widetilde{a}(t, x, \xi)=a(t, x, u(t, x), \xi)$. Hence $u$ is a renormalized solution for $\widetilde{a}$ and the same decomposition of $\mu$. According to [6], the same $u$ is also a renormalized solution for the same $\widetilde{a}$ and any other decomposition of $\mu$. Thus $u$ is a renormalized solution for $a$ and any other decomposition of $\mu$.

Remark 2.6 A renormalized solution to (1.1) is also a solution in the distributional sense. Indeed, if $u$ is a renormalized solution then, by Proposition 4.1, $|u|^{\nu} \in L^{p^{\prime}}(Q)$ and $a(t, x, u, \nabla u)$ is integrable; hence, using $S=S_{n}$ (defined at the beginning of Section 5) in (2.7), we can let $n \rightarrow \infty$ to see, thanks to (2.6), that $u$ satisfies the first equation of (1.1) in the distributional sense.

We now introduce the notion of entropy solution, which is a generalization of the definition given in $[10]$ when $\mu \in L^{1}(Q)$. To this end, we define

$$
E=\left\{\varphi \in L^{p}\left(0, T ; W_{0}^{1, p}(\Omega)\right) \cap L^{\infty}(Q) \mid \varphi_{t} \in L^{p^{\prime}}\left(0, T ; W^{-1, p^{\prime}}(\Omega)\right)+L^{1}(Q)\right\} .
$$

According to [9], one has $E \subset C\left([0, T] ; L^{1}(\Omega)\right)$.

Definition 2.7 Under hypotheses (2.1)-(2.4), if $u_{0} \in L^{1}(\Omega), \mu \in \mathcal{M}_{0}(Q)$ and $\left(f,-\operatorname{div}\left(G_{1}\right), g_{2}\right)$ is a decomposition of $\mu$ according to Theorem 2.2, an entropy solution to (1.1) is a measurable function $u$ such that

$$
T_{k}\left(u-g_{2}\right) \in L^{p}\left(0, T ; W_{0}^{1, p}(\Omega)\right) \text { for all } k \geq 0,
$$

$t \in[0, T] \rightarrow \int_{\Omega} \Theta_{k}\left(u-g_{2}-\varphi\right)(t, x) d x$ is (a.e. equal to) a continuous function,

for all $k \geq 0$ and all $\varphi \in E$,

$$
\begin{gathered}
\int_{\Omega} \Theta_{k}\left(u-g_{2}-\varphi\right)(T, x) d x-\int_{\Omega} \Theta_{k}\left(u_{0}(x)-\varphi(0, x)\right) d x \\
\quad+\int_{0}^{T}\left\langle\varphi_{t}, T_{k}\left(u-g_{2}-\varphi\right)\right\rangle d t
\end{gathered}
$$




$$
\begin{gathered}
+\int_{Q} a(t, x, u, \nabla u) \cdot \nabla\left(T_{k}\left(u-g_{2}-\varphi\right)\right) d t d x \\
\leq \int_{Q} f T_{k}\left(u-g_{2}-\varphi\right) d t d x+\int_{\Omega} G_{1} \cdot \nabla\left(T_{k}\left(u-g_{2}-\varphi\right)\right) d t d x \\
\text { for all } k \geq 0 \text { and all } \varphi \in E
\end{gathered}
$$

Remark 2.8 In (2.11), we denote by $\langle\cdot, \cdot\rangle$ the duality product between $W^{-1, p^{\prime}}(\Omega)$ $+L^{1}(\Omega)$ and $W_{0}^{1, p}(\Omega) \cap L^{\infty}(\Omega)$.

In section 4 , we will prove that each term in (2.11) is well defined.

Remark 2.9 In fact, Definitions 2.3 and 2.7 of renormalized and entropy solutions, and all the results we present here, are also valid for (1.1) with right-hand sides of the kind " $f-\operatorname{div}\left(G_{1}\right)+\partial_{t} g_{2} "$ (with $\left.f \in L^{1}(Q), G_{1} \in L^{p^{\prime}}(Q)\right)^{N}$ and $\left.g_{2} \in L^{p}\left(0, T ; W_{0}^{1, p}(\Omega) \cap L^{2}(\Omega)\right)\right)$, even if they do not come from a measure.

\section{Main results}

The main result of this paper is the following theorem.

Theorem 3.1 Under Hypotheses (2.1)-(2.4), a function is an entropy solution of (1.1) if and only if it is a renormalized solution of (1.1).

The definition we have chosen of entropy solution uses an inequality in (2.11) instead of an equality (such as for renormalized solutions); this is a standard choice for entropy solutions because it is sufficient to obtain the uniqueness (in the case where $a$ does not depend on $u$ and $\mu \in L^{1}(Q)$ for example; see [10]) and makes the proof of the existence quite easier (there is no need to prove the strong convergence of the gradient of the approximate solutions). However, as an immediate corollary of Theorem 3.1 (see Remark 5.2 for the first part and Remark 2.5 for the last one), we also have:

Corollary 3.2 Under Hypotheses (2.1)-(2.4), an entropy solution to (1.1) also satisfies (2.11) with an equality instead of an inequality. Moreover, the definition of entropy solution does not depend on the decomposition of $\mu$ : if a function is an entropy solution for a particular decomposition of $\mu$, then it is an entropy solution for any decomposition of $\mu$.

Remark 3.3 The exponent of $|s|$ in (2.3) may seem curious. It is chosen to ensure that, if $u$ is an entropy or a renormalized solution of (1.1), then $|u|^{\nu} \in$ $L^{p^{\prime}}(Q)$ - we need such a property to prove both implications of Theorem 3.1. Since the regularity property of Proposition 4.1 below is the best we can get (see Remark 4.4), this choice of $\nu$ is also optimal. 
But it is to be noticed that, if we try and use the methods of [6] to prove the existence of a renormalized solution to (1.1) (with $a$ depending on $u$, which was not the case in [6]), then we also need an estimate on $|u|^{\nu}$ in $L^{p^{\prime}}(Q)$ (in particular to prove (2.6)). Thus, this limit on $\nu$ does not seem to be a technical one and to be restricted to the present work, but is also a natural hypothesis when dealing with renormalized solutions to (1.1) in the case where $a$ depends on $u$.

Remark 3.4 Another consequence of Theorem 3.1 is the fact that an entropy solution to (1.1) is also a solution in the distributional sense (see Remark 2.6).

\section{Coherence of the definitions and regularity results}

Let us see that, if (2.5) is satisfied, then (2.7) and (2.8) make sense.

Let $S \in W^{2, \infty}(\mathbf{R})$ such that $S^{\prime}$ has a compact support. We have $u-g_{2} \in$ $L^{\infty}\left(0, T ; L^{1}(\Omega)\right) \subset L^{1}(Q)$, so that, $S$ being Lipschitz-continuous, $S\left(u-g_{2}\right) \in$ $L^{1}(Q)$ and its time-derivative exists in $\mathcal{D}^{\prime}(Q)$. We clearly have

$$
S^{\prime}\left(u-g_{2}\right) f \in L^{1}(Q) \quad \text { and } \quad S^{\prime}\left(u-g_{2}\right) G_{1} \in L^{p^{\prime}}(Q)^{N} .
$$

Taking $M$ a real number such that $\operatorname{supp}\left(S^{\prime}\right) \subset[-M, M]$, we see that, when $S^{\prime}\left(u-g_{2}\right) \neq 0$ or $S^{\prime \prime}\left(u-g_{2}\right) \neq 0$, we have $\left|u-g_{2}\right| \leq M$ so that $u-g_{2}=T_{M}\left(u-g_{2}\right)$ and $\nabla u=\nabla\left(T_{M}\left(u-g_{2}\right)\right)+\nabla g_{2}$ (by definition of $\nabla u$, see Remark 2.4). Hence, when $S^{\prime}\left(u-g_{2}\right)$ or $S^{\prime \prime}\left(u-g_{2}\right)$ are not null, since $\nu \leq p-1$,

$$
\left|\nabla\left(u-g_{2}\right)\right|=\left|\nabla\left(T_{M}\left(u-g_{2}\right)\right)+\nabla g_{2}\right| \in L^{p}(Q)
$$

and

$$
\begin{aligned}
|a(t, x, u, \nabla u)| \leq & \beta\left(b(t, x)+\left|T_{M}\left(u-g_{2}\right)+g_{2}\right|^{\nu}+\left|\nabla\left(T_{M}\left(u-g_{2}\right)\right)+\nabla g_{2}\right|^{p-1}\right) \\
\leq & C \beta\left(b(t, x)+1+\left.|M+| g_{2}\right|^{p-1}+\left|\nabla\left(T_{M}\left(u-g_{2}\right)\right)+\nabla g_{2}\right|^{p-1}\right) \\
& \in L^{p^{\prime}}(Q) .
\end{aligned}
$$

This shows that

$$
\begin{aligned}
& S^{\prime}\left(u-g_{2}\right) a(t, x, u, \nabla u) \in L^{p^{\prime}}(Q)^{N} \\
& S^{\prime \prime}\left(u-g_{2}\right) a(t, x, u, \nabla u) \cdot \nabla\left(u-g_{2}\right) \in L^{1}(Q) \\
& \text { and } S^{\prime \prime}\left(u-g_{2}\right) G_{1} \cdot \nabla\left(u-g_{2}\right) \in L^{1}(Q) .
\end{aligned}
$$

Equations (4.1) and (4.3) prove that each term in (2.7) is defined in $\mathcal{D}^{\prime}(Q)$ and, moreover, that $\left(S\left(u-g_{2}\right)\right)_{t} \in L^{p^{\prime}}\left(0, T ; W^{-1, p^{\prime}}(\Omega)\right)+L^{1}(Q)$. Since $(2.5)$ implies $S\left(u-g_{2}\right) \in L^{p}\left(0, T ; W_{0}^{1, p}(\Omega)\right)$ (because, $S^{\prime}$ being null outside $[-M, M]$, we have $\left.S\left(u-g_{2}\right)=S\left(T_{M}\left(u-g_{2}\right)\right)\right)$, [9] gives then $S\left(u-g_{2}\right) \in C\left([0, T] ; L^{1}(\Omega)\right)$ and (2.8) makes sense. Furthermore, since $\left(S\left(u-g_{2}\right)\right)_{t} \in L^{p^{\prime}}\left(0, T ; W^{-1, p^{\prime}}(\Omega)\right)+L^{1}(Q)$ 
we can use, as test functions in (2.7), not only functions in $C_{c}^{\infty}(Q)$ but also functions in $L^{p}\left(0, T ; W_{0}^{1, p}(\Omega)\right) \cap L^{\infty}(Q)$.

Let us now consider the case of entropy solutions: we want to prove that if $u$ satisfies (2.9) and (2.10), then each term in (2.11) is well defined.

Tanks to (2.10), the first term of (2.11) is well defined; notice that, to be precise, in this term we take the value at $t=T$ of the continuous representative of $t \rightarrow \int_{\Omega} \Theta_{k}\left(u-g_{2}-\varphi\right)(t, x) d x$ (this comes down to taking the essential limit of this function as $t \rightarrow T)$. We have $\left|\Theta_{k}(s)\right| \leq k|s|$; since $u_{0} \in L^{1}(\Omega)$ and $\varphi \in C\left([0, T] ; L^{1}(\Omega)\right)$, we deduce that $\Theta_{k}\left(u_{0}-\varphi(0, \cdot)\right)$ is integrable on $\Omega$ and that the second term of (2.11) makes sense. The third term and the right-hand side are well defined thanks to the regularity on $\varphi_{t}, f$ and $G_{1}$ and to (2.9).

It remains to study the fourth term. On the set $\left\{\nabla T_{k}\left(u-g_{2}-\varphi\right) \neq 0\right\}$, we have $\left|u-g_{2}-\varphi\right| \leq k$, which implies $\left|u-g_{2}\right| \leq M=k+\|\varphi\|_{L^{\infty}(Q)}$. Hence, on this set, (4.2) holds. Since we have, on $Q, T_{k}\left(u-g_{2}-\varphi\right)=T_{k}\left(T_{M}\left(u-g_{2}\right)-\varphi\right) \in$ $L^{p}\left(0, T ; W_{0}^{1, p}(\Omega)\right)$, we deduce that

$$
a(t, x, u, \nabla u) \cdot \nabla\left(T_{k}\left(u-g_{2}-\varphi\right)\right) \in L^{1}(Q)
$$

(notice that this has been obtained under the sole property (2.9) of $u$ ), and all the terms of (2.11) are well defined.

To conclude this subsection, we state and prove a regularity result on entropy and renormalized solutions. This result is mainly useful to bound the term $|u|^{\nu}$ coming from (2.3).

Proposition 4.1 If $u$ is an entropy or a renormalized solution of (1.1), then

i) $u-g_{2} \in L^{\infty}\left(0, T ; L^{1}(\Omega)\right)$,

ii) for all $0 \leq q<p\left(1-\frac{1}{p^{*}}\right),|u|^{q} \in L^{1}(Q)$

iii) for all $0 \leq q<p-\left(1+\frac{1}{p}-\frac{1}{p^{*}}\right)^{-1},|\nabla u|^{q} \in L^{1}(Q)$.

Remark 4.2 Notice that i) gives $u \in L^{1}(Q)$. If $p \leq 2 N /(N+1)$ (hence $N \geq 2$ and $p<N)$, ii) is weaker than i) since $q<\frac{(N+1) p}{N}-1 \leq 1$.

iii) will not be useful to us in the following, but since it is very easy (and classical) to obtain, we state it. If $p<N$, the condition in iii) reduces to $0 \leq q<$ $p-\frac{N}{N+1}$ and, if $p \geq N$, it reduces to $0 \leq q<\frac{p^{2}}{p+1}$.

Remark 4.3 These regularities are compatible with the results of [4]. But we cannot directly use this reference since we have not supposed that all entropy or renormalized solutions are obtained via approximation techniques.

Remark 4.4 These results are the best we can get, in the sense that, in general, we cannot prove that $|u|^{p\left(1-1 / p^{*}\right)} \in L^{1}(Q)$ and $|\nabla u|^{p-\left(1+1 / p-1 / p^{*}\right)^{-1}} \in L^{1}(Q)$. 
Indeed, let $N>p>2 N /(N+1), q=(p-1+p / N)^{\prime}, r=(1+1 /(p-1)(N+1))^{\prime}$, $\beta=N /(q-1), 0<\alpha<\inf (1-1 / q, 1-1 / r)$ and $x_{0} \in \Omega$; then $\varphi(t, x)=\mid \ln (\mid t-$ $\left.T / 2|+| x-\left.x_{0}\right|^{\beta}\right)\left.\right|^{\alpha}$ (truncated away from $\left(T / 2, x_{0}\right)$ ) does not belong to $L^{\infty}(Q)$ but satisfies $\partial_{t} \varphi \in L^{q}(Q)$ and $\nabla_{x} \varphi \in L^{r}(Q)^{N}$. Let $\mu=\sum_{n \geq 1} \frac{1}{n^{2}\left|E_{n}\right|} \mathbf{1}_{E_{n}}$ where $E_{n}=\{n \leq \varphi<n+1\}$ : we have $\mu \in L^{1}(Q)$. Since $\varphi \notin L^{\infty}(Q), \int_{Q} \varphi \mu=+\infty$. Hence $\left.\mu \in L^{1}(Q) \backslash\left[\partial_{t}\left(L^{p-1+p / N}(Q)\right)+\operatorname{div}\left(L^{1+1 /(p-1)(N+1)}(Q)^{N}\right)\right)\right]$.

Let $u$ be the solution to (1.1) with this $\mu$ as data (and supposing that $a$ does not depend on $u$ ). If $|u|^{p\left(1-1 / p^{*}\right)} \in L^{1}(Q)$ and $|\nabla u|^{p-\left(1+1 / p-1 / p^{*}\right)^{-1}} \in L^{1}(Q)$, this would lead to $u \in L^{p-1+p / N}(Q)$ and $\nabla u \in L^{p-N /(N+1)}(Q)^{N}$ which, thanks to $(2.3)$, gives $a(t, x, \nabla u) \in L^{1+1 /(p-1)(N+1)}(Q)^{N}$. Since an entropy or a renormalized solution is also a solution of (1.1) in the sense of distributions (see Remarks 2.6 and 3.4), this would give $\mu \in \partial_{t}\left(L^{p-1+p / N}(Q)\right)+\operatorname{div}\left(L^{1+1 /(p-1)(N+1)}(Q)^{N}\right)$, which is a contradiction.

Remark 4.5 We will prove in fact, as it is usual in elliptic and parabolic problems with measure data, that $u$ and its gradient respectively belong to the Marcinkiewicz spaces of exponents $p\left(1-\frac{1}{p^{*}}\right)$ and $p-\left(1+\frac{1}{p}-\frac{1}{p^{*}}\right)^{-1}$.

\section{Proof of Proposition 4.1}

We let $v=u-g_{2}$, and let $C$ be a constant that may change from line to line but that is not depending on $k$ or $\lambda$.

Step 1: we prove i).

This regularity is in the definition of renormalized solutions.

For entropy solutions, this regularity comes from (2.10). Indeed, take $\varphi=0$ and $k=1$ in this property. The function $t \in[0, T] \rightarrow \int_{\Omega} \Theta_{1}(v)(t, x) d x$ being a.e. equal to a continuous function, it is essentially bounded, say by $M$. Since $\Theta_{1}(s) \geq|s|-1$, we deduce that, for a.e. $t \in[0, T]$,

$$
\begin{aligned}
& \int_{\Omega}|v|(t, x) d x \\
& \quad \leq \int_{\Omega}\left(1+\Theta_{1}(v)(t, x)\right) d x \leq \operatorname{meas}(\Omega)+M
\end{aligned}
$$

which proves that $v \in L^{\infty}\left(0, T ; L^{1}(\Omega)\right)$.

To prove ii), we use classical techniques for elliptic or parabolic equations with measure data.

Step 2: an estimate on $\nabla\left(T_{k}(v)\right)$.

We first handle the case of entropy solutions. Using $\varphi=0$ in (2.11) for some $k \geq 0$, and since $\Theta_{k} \geq 0$, we see that

$$
\begin{aligned}
& \int_{Q} a(t, x, u, \nabla u) \cdot \nabla\left(T_{k}(v)\right) d t d x \\
& \quad \leq \int_{\Omega} \Theta_{k}\left(u_{0}(x)\right) d x+k\|f\|_{L^{1}(Q)}+\left\|G_{1}\right\|_{L^{p^{\prime}}(Q)}\left\|\nabla\left(T_{k}(v)\right)\right\|_{L^{p}(Q)} .
\end{aligned}
$$


But, since $\left|T_{k}(s)\right| \leq k$, we have $\Theta_{k}(s) \leq k|s|$, so that

$$
\begin{aligned}
\int_{Q} a(t, x, u, \nabla u) \cdot \nabla\left(T_{k}(v)\right) d t d x \leq & k\left\|u_{0}\right\|_{L^{1}(\Omega)}+k\|f\|_{L^{1}(Q)} \\
& +\left\|G_{1}\right\|_{L^{p^{\prime}}(Q)}\left\|\nabla\left(T_{k}(v)\right)\right\|_{L^{p}(Q)} .
\end{aligned}
$$

Recall that $v=u-g_{2}$. By (2.2) and (2.3) and since $\nu \leq p-1$, we have, on $\{|v| \leq k\}$

$$
\begin{aligned}
& a(t, x, u, \nabla u) \cdot \nabla\left(T_{k}(v)\right) \\
& \quad \geq \alpha|\nabla u|^{p}-\Lambda(t, x)-\beta\left(b(t, x)+|u|^{\nu}+|\nabla u|^{p-1}\right)\left|\nabla g_{2}\right| \\
& \quad \geq \alpha|\nabla u|^{p}-\Lambda(t, x)-\beta\left(\tilde{b}(t, x)+|u|^{p-1}+|\nabla u|^{p-1}\right)\left|\nabla g_{2}\right|
\end{aligned}
$$

where $\tilde{b}=1+b$. Since $\nabla T_{k}(v)=0$ outside $\{|v| \leq k\}$, we deduce

$$
\begin{aligned}
& \alpha \int_{\{|v| \leq k\}}|\nabla u|^{p} d t d x \\
& \quad \leq \int_{Q} a(t, x, u, \nabla u) \cdot \nabla\left(T_{k}(v)\right) d t d x+\int_{Q} \Lambda d t d x \\
& \quad+\beta\left\|\nabla g_{2}\right\|_{L^{p}(Q)}\left(\|\tilde{b}\|_{L^{p^{\prime}(Q)}}+\|u\|_{L^{p}(\{|v| \leq k\})}^{p-1}+\|\nabla u\|_{L^{p}(\{|v| \leq k\})}^{p-1}\right) .
\end{aligned}
$$

On $\{|v| \leq k\}$, we have $u=T_{k}(v)+g_{2}$ and $\nabla u=\nabla\left(T_{k}(v)\right)+\nabla g_{2}$; therefore, we can find $C$ not depending on $k$ such that

$$
\begin{aligned}
& \alpha \int_{\{|v| \leq k\}}|\nabla u|^{p} d t d x \\
& \quad \leq \int_{Q} a(t, x, u, \nabla u) \cdot \nabla\left(T_{k}(v)\right) d t d x+\int_{Q} \Lambda d t d x \\
& \quad+C\left(1+\left\|T_{k}(v)\right\|_{L^{p}(Q)}^{p-1}+\left\|g_{2}\right\|_{L^{p}(Q)}^{p-1}+\left\|\nabla\left(T_{k}(v)\right)\right\|_{L^{p}(Q)}^{p-1}+\left\|\nabla g_{2}\right\|_{L^{p}(Q)}^{p-1}\right) .
\end{aligned}
$$

Using Poincaré's inequality, we finally obtain

$$
\begin{aligned}
& \alpha \int_{\{|v| \leq k\}}|\nabla u|^{p} d t d x \\
& \quad \leq \int_{Q} a(t, x, u, \nabla u) \cdot \nabla\left(T_{k}(v)\right) d t d x+C\left(1+\left\|\nabla\left(T_{k}(v)\right)\right\|_{L^{p}(Q)}^{p-1}\right)
\end{aligned}
$$

(we have also put $\int_{Q} \Lambda$ in $C$ ). Now, we write

$$
\begin{aligned}
\int_{Q} & \left|\nabla\left(T_{k}(v)\right)\right|^{p} d t d x \\
\quad= & \int_{\{|v| \leq k\}}\left|\nabla\left(u-g_{2}\right)\right|^{p} d t d x \leq C \int_{\{|v| \leq k\}}|\nabla u|^{p} d t d x+C \int_{Q}\left|\nabla g_{2}\right|^{p} d t d x
\end{aligned}
$$


so that (4.5) and (4.6) give

$$
\int_{Q}\left|\nabla\left(T_{k}(v)\right)\right|^{p} d t d x \leq C+C\left\|\nabla\left(T_{k}(v)\right)\right\|_{L^{p}(Q)}^{p-1}+C k+C\left\|\nabla\left(T_{k}(v)\right)\right\|_{L^{p}(Q)} .
$$

But Young's inequality allows to write

$$
\left\|\nabla\left(T_{k}(v)\right)\right\|_{L^{p}(Q)}^{p-1} \leq C+\frac{1}{4}\left\|\nabla\left(T_{k}(v)\right)\right\|_{L^{p}(Q)}^{p}
$$

and

$$
\left\|\nabla\left(T_{k}(v)\right)\right\|_{L^{p}(Q)} \leq C+\frac{1}{4}\left\|\nabla\left(T_{k}(v)\right)\right\|_{L^{p}(Q)}^{p} .
$$

We thus get, for all $k \geq 1$,

$$
\int_{Q}\left|\nabla\left(T_{k}(v)\right)\right|^{p} d t d x \leq C k .
$$

For renormalized solutions, this estimate is very easy to obtain. We have

$$
\begin{gathered}
\int_{Q}\left|\nabla\left(T_{k}(v)\right)\right|^{p} d t d x=\int_{\{|v|<k\}}\left|\nabla u-\nabla g_{2}\right|^{p} d t d x \\
\leq C \int_{\{|v|<k\}}|\nabla u|^{p} d t d x+C \int_{Q}\left|\nabla g_{2}\right|^{p} d t d x .
\end{gathered}
$$

But, by (2.5) and (2.6), $\left(\int_{\{n \leq|v|<n+1\}}|\nabla u|^{p}\right)_{n \geq 0}$ is bounded (say by $M$ ); therefore, for $k \geq 1$ and denoting by $[k]$ the integer part of $k$,

$$
\int_{\{|v| \leq k\}}|\nabla u|^{p} d t d x \leq \sum_{n=0}^{[k]} \int_{\{n \leq|v|<n+1\}}|\nabla u|^{p} d t d x \leq M([k]+1) \leq M(k+2) .
$$

By (4.9), we deduce that (4.7) holds for all $k \geq 1$.

Step 3: conclusion $\left({ }^{1}\right)$.

Let $\theta$ and $r$ satisfy $\frac{1}{r}=\frac{1-\theta}{\infty}+\frac{\theta}{p}=\frac{1-\theta}{1}+\frac{\theta}{p^{*}}$; this means that $\theta\left(1+\frac{1}{p}-\frac{1}{p^{*}}\right)=1$, which defines $\theta \in[0,1]$ (because $\left.\frac{1}{p} \geq \frac{1}{p^{*}}\right)$, and that $r=\frac{p}{\theta}=1+p\left(1-\frac{1}{p^{*}}\right) \in[1, \infty[$. Hence, some easy interpolations in Lebesgue spaces give

$$
\left\|T_{k}(v)\right\|_{L^{r}(Q)}=\left\|T_{k}(v)\right\|_{L^{r}\left(0, T ; L^{r}(\Omega)\right)} \leq\left\|T_{k}(v)\right\|_{L^{\infty}\left(0, T ; L^{1}(\Omega)\right)}^{1-\theta}\left\|T_{k}(v)\right\|_{L^{p}\left(0, T ; L^{p^{*}}(\Omega)\right)}^{\theta} .
$$

Using the fact that $v \in L^{\infty}\left(0, T ; L^{1}(\Omega)\right)$ and $\left|T_{k}(v)\right| \leq|v|$, we see that $\left\|T_{k}(v)\right\|_{L^{\infty}\left(0, T ; L^{1}(\Omega)\right)}^{1-\theta}$ is bounded by $C$ (not depending on $k \geq 1$ ). By Sobolev's injection, (4.7) shows that, if $k \geq 1$,

$$
\left\|T_{k}(v)\right\|_{L^{p}\left(0, T ; L^{p^{*}}(\Omega)\right)}^{\theta} \leq C\left\|T_{k}(v)\right\|_{L^{p}\left(0, T ; W_{0}^{1, p}(\Omega)\right)}^{\theta} \leq C k^{\theta / p} .
$$

\footnotetext{
${ }^{1}$ The deduction of ii) and iii) from i) and (4.7) is adapted from e.g. [4] or [1].
} 
Hence, we get

$$
\left\|T_{k}(v)\right\|_{L^{r}(Q)} \leq C k^{\theta / p}=C k^{1 / r} .
$$

This shows, by Tchebycheff's inequality, that meas $(\{|v| \geq k\})=\operatorname{meas}\left(\left\{\left|T_{k}(v)\right| \geq\right.\right.$ $k\}) \leq C k^{-(r-1)}$, i.e. that $v$ belongs to $M^{r-1}$, the Marcinkiewicz space of exponent $r-1$.

For $\lambda \geq 0$ and $k \geq 1$, we can write

$$
\{|\nabla v| \geq k\} \subset\{|v| \geq \lambda\} \cup\left\{\left|\nabla\left(T_{\lambda}(v)\right)\right| \geq k\right\} \cup A
$$

where $\operatorname{meas}(A)=0$ (this comes from the fact that $\nabla v=\nabla\left(T_{\lambda}(v)\right.$ ) a.e. on $\{|v| \leq \lambda\}$ ). Thus, applying Tchebycheff's inequality to (4.7) (with $\lambda$ instead of $k$ ) and using the fact that $v \in M^{r-1}$, there exists $C$ (not depending on $\lambda$ nor $k$ ) such that

$$
\operatorname{meas}(\{|\nabla v| \geq k\}) \leq C \lambda^{1-r}+C \lambda k^{-p} .
$$

Minimizing this inequality on $\lambda$ we see that an optimal choice is, up to a multiplicative constant, $\lambda=k^{p / r}=k^{\theta}$, which leads to meas $(\{|\nabla v| \geq k\}) \leq C k^{-(p-\theta)}$. Hence, $\nabla v \in M^{p-\theta}$.

Since $v \in M^{r-1}$ and $\nabla v \in M^{p-\theta}$, classical integration properties of functions in Marcinkiewicz spaces imply that, for all $0 \leq q<r-1,|v|^{q} \in L^{1}(Q)$ and that, for all $0 \leq q<p-\theta,|\nabla v|^{q} \in L^{1}(Q)$.

We have $\theta=\left(1+\frac{1}{p}-\frac{1}{p^{*}}\right)^{-1}$ and $r-1=p\left(1-\frac{1}{p^{*}}\right)$. Moreover, for all $0 \leq q<$ $p\left(1-\frac{1}{p^{*}}\right) \leq p$, we have $\left|g_{2}\right|^{q} \in L^{1}(Q)$ and for all $0 \leq q<p-\left(1+\frac{1}{p}-\frac{1}{p^{*}}\right)^{-1} \leq p$, $\left|\nabla g_{2}\right|^{q} \in L^{1}(Q)$ (because $\left.g_{2} \in L^{p}\left(0, T ; W_{0}^{1, p}(\Omega)\right)\right)$.

Since $u=v+g_{2}$ and $\nabla u=\nabla v+\nabla g_{2}$, we deduce therefore that, for all $0 \leq q<p\left(1-\frac{1}{p^{*}}\right),|u|^{q} \leq C\left(|v|^{q}+\left|g_{2}\right|^{q}\right) \in L^{1}(Q)$ and that, for all $0 \leq q<$ $p-\left(1+\frac{1}{p}-\frac{1}{p^{*}}\right)^{-1},|\nabla u|^{q} \leq C\left(|\nabla v|^{q}+\left|\nabla g_{2}\right|^{q}\right) \in L^{1}(Q)$. This concludes the proof of ii) and iii).

\section{A renormalized solution is an entropy solution}

In the following, we make a constant use of the function $S_{n}: \mathbf{R} \rightarrow \mathbf{R}$ defined by:

$$
h_{n}(s)=1-\left|T_{1}\left(s-T_{n}(s)\right)\right|, \quad S_{n}(s)=\int_{0}^{s} h_{n}(r) d r .
$$

It satisfies $S_{n}^{\prime \prime}=\mathbf{1}_{[-n-1,-n]}-\mathbf{1}_{[n, n+1]}$.

We prove in this section that if $u$ is a renormalized solution to (1.1), then it is also an entropy solution.

Property (2.9) of the entropy solution is contained in (2.5). Thus, it remains to prove that $u$ satisfies $(2.10)$ and $(2.11)$; this is done simultaneously.

Let $\left.\left.t_{1} \in\right] 0, T\right]$ and $\theta_{\varepsilon}(t)=1-\left(\left(t-t_{1}\right)^{+} / \varepsilon\right)$, i.e. $\theta_{\varepsilon}$ is continuous on $[0, \infty[$, $\theta_{\varepsilon}=1$ on $\left[0, t_{1}\right], \theta_{\varepsilon}=0$ on $\left[t_{1}+\varepsilon, \infty\left[\right.\right.$ and $\theta_{\varepsilon}$ is linear on $\left[t_{1}, t_{1}+\varepsilon\right]$. We denote $v=u-g_{2}$. 
Let $\varphi \in E$ and $M=k+\|\varphi\|_{L^{\infty}(Q)}$; we have $\varphi \in C\left([0, T] ; L^{1}(\Omega)\right)$. The function $T_{k}(v-\varphi) \theta_{\varepsilon}=T_{k}\left(T_{M}(v)-\varphi\right) \theta_{\varepsilon}$ is in $L^{p}\left(0, T ; W_{0}^{1, p}(\Omega)\right) \cap L^{\infty}(Q)$ (using $(2.5))$ and we can thus use it as a test function in (2.7):

$$
\begin{aligned}
\int_{0}^{T} & \theta_{\varepsilon}\left\langle\left(S_{n}(v)\right)_{t}, T_{k}(v-\varphi)\right\rangle d t+\int_{Q} \theta_{\varepsilon} S_{n}^{\prime}(v) a(t, x, u, \nabla u) \cdot \nabla\left(T_{k}(v-\varphi)\right) d t d x \\
= & \int_{Q} \theta_{\varepsilon} S_{n}^{\prime}(v) f T_{k}(v-\varphi) d t d x+\int_{Q} \theta_{\varepsilon} S_{n}^{\prime}(v) G_{1} \cdot \nabla\left(T_{k}(v-\varphi)\right) d t d x \\
& -\int_{Q} \theta_{\varepsilon} S_{n}^{\prime \prime}(v) a(t, x, u, \nabla u) \cdot \nabla v T_{k}(v-\varphi) d t d x \\
& +\int_{Q} \theta_{\varepsilon} S_{n}^{\prime \prime}(v) G_{1} \cdot \nabla v T_{k}(v-\varphi) d t d x
\end{aligned}
$$

We have $\left(S_{n}(v)\right)_{t} \in L^{p^{\prime}}\left(0, T ; W^{-1, p^{\prime}}(\Omega)\right)+L^{1}(Q)$ (see Section 4) and $T_{k}(v-\varphi) \in L^{p}\left(0, T ; W_{0}^{1, p}(\Omega)\right) \cap L^{\infty}(Q)$; hence, $\left\langle\left(S_{n}(v)\right)_{t}, T_{k}(v-\varphi)\right\rangle \in L^{1}(0, T)$. We also have $a(t, x, u, \nabla u) \cdot \nabla\left(T_{k}(v-\varphi)\right) \in L^{1}(Q)$ (see the proof of (4.4)), $f T_{k}(v-\varphi) \in L^{1}(Q)$ and $G_{1} \cdot \nabla\left(T_{k}(v-\varphi)\right)=G_{1} \cdot \nabla\left(T_{k}\left(T_{M}(v)-\varphi\right)\right) \in L^{1}(Q)$. Moreover, since $S_{n}^{\prime \prime}(s)=0$ for $|s| \notin[n, n+1]$, we can write

$$
\begin{aligned}
& S_{n}^{\prime \prime}(v) a(t, x, u, \nabla u) \cdot \nabla v T_{k}(v-\varphi) \\
& \quad=S_{n}^{\prime \prime}(v) a(t, x, u, \nabla u) \cdot \nabla\left(T_{n+1}(v)\right) T_{k}(v-\varphi) \in L^{1}(Q)
\end{aligned}
$$

(we also use (4.4) here, but with $\varphi=0$ and $n+1$ instead of $k$ ) and

$$
S_{n}^{\prime \prime}(v) G_{1} \cdot \nabla v T_{k}(v-\varphi)=S_{n}^{\prime \prime}(v) G_{1} \cdot \nabla\left(T_{n+1}(v)\right) T_{k}(v-\varphi) \in L^{1}(Q) .
$$

Since $\theta_{\varepsilon} \rightarrow \mathbf{1}_{\left[0, t_{1}\right]}$ and is bounded by 1 as $\varepsilon \rightarrow 0$, the dominated convergence theorem therefore gives, with $Q_{t_{1}}=\left[0, t_{1}\right] \times \Omega$,

$$
\begin{aligned}
\int_{0}^{t_{1}} & \left\langle\left(S_{n}(v)\right)_{t}, T_{k}(v-\varphi)\right\rangle d t+\int_{Q_{t_{1}}} S_{n}^{\prime}(v) a(t, x, u, \nabla u) \cdot \nabla\left(T_{k}(v-\varphi)\right) d t d x \\
= & \int_{Q_{t_{1}}} S_{n}^{\prime}(v) f T_{k}(v-\varphi) d t d x+\int_{Q_{t_{1}}} S_{n}^{\prime}(v) G_{1} \cdot \nabla\left(T_{k}(v-\varphi)\right) d t d x \\
& -\int_{Q_{t_{1}}} S_{n}^{\prime \prime}(v) a(t, x, u, \nabla u) \cdot \nabla v T_{k}(v-\varphi) d t d x \\
& +\int_{Q_{t_{1}}} S_{n}^{\prime \prime}(v) G_{1} \cdot \nabla v T_{k}(v-\varphi) d t d x .
\end{aligned}
$$

Let $n \geq M$. We have $T_{k}(v-\varphi)=T_{k}\left(S_{n}(v)-\varphi\right)$ (since $S_{n}(s)=s$ on $[-M, M]$, and $\left|S_{n}(s)\right| \geq M$ and $\operatorname{sgn}\left(S_{n}(s)\right)=\operatorname{sgn}(s)$ outside $\left.[-M, M]\right)$. We know that $S_{n}(v):[0, T] \rightarrow L^{1}(\Omega)$ is continuous or, more precisely, that $S_{n}(v)$ is a.e. equal on $[0, T]$ to a continuous function $\overline{S_{n}(v)}:[0, T] \rightarrow L^{1}(\Omega)$. Since 
$S_{n}(v)-\varphi \in L^{p}\left(0, T ; W_{0}^{1, p}(\Omega)\right)$ with a time derivative in $L^{p^{\prime}}\left(0, T ; W^{-1, p^{\prime}}(\Omega)\right)+$ $L^{1}(Q)$ (see Section 4 ), we can integrate by parts (using Lemma 7.1 in the appendix) to find, by $(2.8)$,

$$
\begin{aligned}
\int_{0}^{t_{1}} & \left\langle\left(S_{n}(v)\right)_{t}, T_{k}(v-\varphi)\right\rangle d t \\
= & \int_{0}^{t_{1}}\left\langle\left(S_{n}(v)-\varphi\right)_{t}, T_{k}\left(S_{n}(v)-\varphi\right)\right\rangle d t+\int_{0}^{t_{1}}\left\langle\varphi_{t}, T_{k}(v-\varphi)\right\rangle d t \\
= & \int_{\Omega} \Theta_{k}\left(\overline{S_{n}(v)}\left(t_{1}\right)-\varphi\left(t_{1}\right)\right) d x-\int_{\Omega} \Theta_{k}\left(S_{n}\left(u_{0}\right)-\varphi(0)\right) d x \\
& +\int_{0}^{t_{1}}\left\langle\varphi_{t}, T_{k}(v-\varphi)\right\rangle d t
\end{aligned}
$$

and we deduce from (5.2) that

$$
\begin{aligned}
\int_{\Omega} \Theta_{k}\left(\overline{S_{n}(v)}\left(t_{1}\right)-\varphi\left(t_{1}\right)\right) d x-\int_{\Omega} \Theta_{k}\left(S_{n}\left(u_{0}\right)-\varphi(0)\right) d x \\
\quad+\int_{0}^{t_{1}}\left\langle\varphi_{t}, T_{k}(v-\varphi)\right\rangle d t+\int_{Q_{t_{1}}} S_{n}^{\prime}(v) a(t, x, u, \nabla u) \cdot \nabla\left(T_{k}(v-\varphi)\right) d t d x \\
=\int_{Q_{t_{1}}} S_{n}^{\prime}(v) f T_{k}(v-\varphi) d t d x+\int_{Q_{t_{1}}} S_{n}^{\prime}(v) G_{1} \cdot \nabla\left(T_{k}(v-\varphi)\right) d t d x \\
\quad-\int_{Q_{t_{1}}} S_{n}^{\prime \prime}(v) a(t, x, u, \nabla u) \cdot \nabla v T_{k}(v-\varphi) d t d x \\
\quad+\int_{Q_{t_{1}}} S_{n}^{\prime \prime}(v) G_{1} \cdot \nabla v T_{k}(v-\varphi) d t d x .
\end{aligned}
$$

We now want to let $n \rightarrow \infty$, with a good choice of $t_{1}$. Let $(A)-(H)$ be the terms in this equality.

For all $n \geq 1$, we have $\overline{S_{n}(v)}=S_{n}(v)$ a.e. on $Q$; since these are a countable number of a.e. equalities, we can suppose that they are all satisfied outside a common subset of $Q$ of null measure. Thus, for a.e. $t_{1} \in[0, T]$, we have, a.e. on $\Omega, \overline{S_{n}(v)}\left(t_{1}, \cdot\right)-\varphi\left(t_{1}, \cdot\right)=S_{n}\left(v\left(t_{1}, \cdot\right)\right)-\varphi\left(t_{1}, \cdot\right) \rightarrow v\left(t_{1}, \cdot\right)-\varphi\left(t_{1}, \cdot\right)$ as $n \rightarrow \infty$ and $\left|\overline{S_{n}(v)}\left(t_{1}, \cdot\right)-\varphi\left(t_{1}, \cdot\right)\right| \leq\left|v\left(t_{1}, \cdot\right)\right|+\left|\varphi\left(t_{1}, \cdot\right)\right| \in L^{1}(\Omega)$ (recall that $\varphi \in C\left([0, T] ; L^{1}(\Omega)\right)$ and that $v \in L^{\infty}\left(0, T ; L^{1}(\Omega)\right)$ by $\left.(2.5)\right)$. The dominated convergence theorem therefore shows that, for a.e. $t_{1} \in[0, T], \overline{S_{n}(v)}\left(t_{1}, \cdot\right)-\varphi\left(t_{1}, \cdot\right) \rightarrow$ $v\left(t_{1}, \cdot\right)-\varphi\left(t_{1}, \cdot\right)$ in $L^{1}(\Omega)$ as $n \rightarrow \infty ; \Theta_{k}$ being Lipschitz-continuous, we deduce that, for a.e. $t_{1} \in[0, T],(A)$ tends to $\int_{\Omega} \Theta_{k}(v-\varphi)\left(t_{1}\right) d x$.

By dominated convergence we have $S_{n}\left(u_{0}\right) \rightarrow u_{0}$ in $L^{1}(\Omega)$ and since $\Theta_{k}$ is Lipschitz-continuous, we see that $(B)$ tends to $-\int_{\Omega} \Theta_{k}\left(u_{0}-\varphi(0)\right) d x$ as $n \rightarrow \infty$. $(C)$ does not depend on $n$. As we have seen before, $a(t, x, u, \nabla u) \cdot \nabla\left(T_{k}(v-\varphi)\right)$, $f T_{k}(v-\varphi)$ and $G_{1} \cdot \nabla\left(T_{k}(v-\varphi)\right)$ all belong to $L^{1}(Q)$; since $S_{n}^{\prime}(v) \rightarrow 1$ and $\left|S_{n}^{\prime}\right| \leq 1$, we deduce from the dominated convergence theorem that $(D) \rightarrow$ 
$\int_{Q_{t_{1}}} a(t, x, u, \nabla u) \cdot \nabla\left(T_{k}(v-\varphi)\right) d t d x$, that $(E) \rightarrow \int_{Q_{t_{1}}} f T_{k}(v-\varphi) d t d x$ and that $(F) \rightarrow \int_{Q_{t_{1}}} G_{1} \cdot \nabla\left(T_{k}(v-\varphi)\right) d t d x$ as $n \rightarrow \infty$.

Since $\left|S_{n}^{\prime \prime}\right| \leq 1$ and $S_{n}^{\prime \prime}(s) \neq 0$ only if $|s| \in[n, n+1]$, we can write, with $C$ not depending on $n$ (and that may change from line to line),

$$
\begin{aligned}
|(\mathrm{G})|+|(\mathrm{H})| & \leq C \int_{\{n \leq|v| \leq n+1\}}\left(b+|u|^{\nu}+|\nabla u|^{p-1}\right)|\nabla v|+\left|G_{1}\right||\nabla v| d t d x \\
& \leq C \int_{\{n \leq|v| \leq n+1\}}\left(b^{p^{\prime}}+|u|^{\nu p^{\prime}}+|\nabla u|^{p}+|\nabla v|^{p}+\left|G_{1}\right|^{p^{\prime}}\right) d t d x \\
& \leq C \int_{\{n \leq|v| \leq n+1\}}\left(b^{p^{\prime}}+|u|^{\nu p^{\prime}}+|\nabla u|^{p}+\left|\nabla g_{2}\right|^{p}+\left|G_{1}\right|^{p^{\prime}}\right) d t d x,
\end{aligned}
$$

(we have used $|\nabla v|^{p} \leq C\left(|\nabla u|^{p}+\left|\nabla g_{2}\right|^{p}\right)$ ); by Proposition 4.1, we have $|u|^{\nu p^{\prime}} \in$ $L^{1}(Q)$ (because $\left.\nu p^{\prime}<p\left(1-\frac{1}{p^{*}}\right)\right)$. Using $(2.6)$ and the fact that meas $(\{n \leq|v| \leq$ $n+1\}) \rightarrow 0$ ( $v$ is a.e. finite), we deduce that $|(G)|+|(H)| \rightarrow 0$ as $n \rightarrow \infty$.

Passing to the limit in (5.3), we obtain, for a.e. $t_{1} \in[0, T]$,

$$
\begin{aligned}
\int_{\Omega} & \Theta_{k}(v-\varphi)\left(t_{1}\right) d x-\int_{\Omega} \Theta_{k}\left(u_{0}-\varphi(0)\right) d x \\
& \quad+\int_{0}^{t_{1}}\left\langle\varphi_{t}, T_{k}(v-\varphi)\right\rangle d t+\int_{Q_{t_{1}}} a(t, x, u, \nabla u) \cdot \nabla\left(T_{k}(v-\varphi)\right) d t d x \\
= & \int_{Q_{t_{1}}} f T_{k}(v-\varphi) d t d x+\int_{Q_{t_{1}}} G_{1} \cdot \nabla\left(T_{k}(v-\varphi)\right) d t d x .
\end{aligned}
$$

The last five terms of this equality are continuous with respect to $t_{1}$ (the second term does not depend on $t_{1}$ and the other ones are integrals signs on $] 0, t_{1}$ [ of functions which are integrable on $[0, T]$ ). This shows that the first term is a.e. equal to a continuous function on $[0, T]$, hence establishing (2.10), and, either taking the essential limit as $t_{1} \rightarrow T$ or replacing the first term by its continuous representative (and then letting $t_{1} \rightarrow T$ ), that (2.11) holds.

Remark 5.1 The distinction between $\overline{S_{n}(v)}$ and $S_{n}(v)$ in the preceding proof may appear exaggerated: we know that $S_{n}(v)$ is (a.e. equal to) a continuous function $[0, T] \rightarrow L^{1}(\Omega)$ so we could directly use this property without dealing with the "a.e.". However, this would lead to a lack of rigor: if we change $S_{n}(v)$ on a set of null measure to ensure that it is continuous, then it is not obvious that we can still write this function as $S_{n}(\bar{v})$ (for some $\bar{v}$ a.e. equal to $v$ and independant of $n$ ); and such a writing (the composite of $S_{n}$ with some function) is vital in order to let $n \rightarrow \infty$ in $(A)$. This is why, though it is quite exceptional, we have to make a distinction between $S_{n}(v)$ and its continuous representative.

Remark 5.2 Notice that we have proved that a renormalized solution satisfies (2.11) not only with an inequality but in fact with an equality. This is something 
that we could have expected: in order to prove the existence of a renormalized solution, on the contrary to the entropy solution, we have to prove a strong convergence of the gradients of the approximate solutions; it is this strong convergence of the gradients that transforms the inequality in (2.11) into an equality (if, in the construction of entropy solutions, we prove the strong convergence of the gradients, then equality in (2.11) naturally appears).

\section{An entropy solution is a renormalized solution}

We now take an entropy solution $u$ and we prove that it is a renormalized solution. We first prove (in Subsection 6.1) that $u$ satisfies (2.6) and then, comparing $u$ to the renormalized solution $\widetilde{u}$ of a modified version of (1.1), that $u$ satisfies all other properties of renormalized solutions (in Subsection 6.2).

\subsection{The integrability property (2.6)}

Let $u$ be an entropy solution and $v=u-g_{2}$. We prove here that, for all $k \geq 0$,

$$
\lim _{h \rightarrow \infty} \int_{\{h \leq|v| \leq h+k\}}|\nabla u|^{p} d t d x=0 .
$$
is to say

Let $h>0$ and $\left(T_{h}(v)\right)_{\zeta}$ be the Landes regularization of $T_{h}(v)$ (see [7]), that

$$
\begin{aligned}
& \left(\left(T_{h}(v)\right)_{\zeta}\right)_{t}=\zeta\left(T_{h}(v)-\left(T_{h}(v)\right)_{\zeta}\right), \quad\left(T_{h}(v)\right)_{\zeta}(0)=z_{\zeta}, \\
& \text { with } z_{\zeta} \in W_{0}^{1, p}(\Omega),\left\|z_{\zeta}\right\|_{L^{\infty}(\Omega)} \leq h, z_{\zeta} \rightarrow T_{h}\left(u_{0}\right) \text { in } L^{1}(\Omega) \text { as } \zeta \rightarrow \infty \\
& \text { and } \frac{1}{\zeta}\left\|z_{\zeta}\right\|_{W_{0}^{1, p}(\Omega)} \rightarrow 0 \text { as } \zeta \rightarrow \infty .
\end{aligned}
$$

It is known that $\left|\left(T_{h}(v)\right)_{\zeta}\right| \leq h$ and that, up to a subsequence, $\left(T_{h}(v)\right)_{\zeta} \rightarrow T_{h}(v)$ in $L^{p}\left(0, T ; W_{0}^{1, p}(\Omega)\right)$ and a.e. on $Q$ as $\zeta \rightarrow \infty$.

$\left(T_{h}(v)\right)_{\zeta}$ belongs to $L^{p}\left(0, T ; W_{0}^{1, p}(\Omega)\right) \cap L^{\infty}(Q)$ and $\left(\left(T_{h}(v)\right)_{\zeta}\right)_{t}=\zeta\left(T_{h}(v)-\right.$ $\left.\left(T_{h}(v)\right)_{\zeta}\right)$ is in $L^{\infty}(Q) \subset L^{p^{\prime}}\left(0, T ; W^{-1, p^{\prime}}(\Omega)\right)+L^{1}(Q)$. Hence, we can use it as a test function in (2.11) to find

$$
\begin{aligned}
& \int_{\Omega} \Theta_{k}\left(v-\left(T_{h}(v)\right)_{\zeta}\right)(T) d x-\int_{\Omega} \Theta_{k}\left(u_{0}-z_{\zeta}\right) d x \\
& \quad+\int_{0}^{T}\left\langle\left(\left(T_{h}(v)\right)_{\zeta}\right)_{t}, T_{k}\left(v-\left(T_{h}(v)\right)_{\zeta}\right)\right\rangle d t \\
& \quad+\int_{Q} a(t, x, u, \nabla u) \cdot \nabla\left(T_{k}\left(v-\left(T_{h}(v)\right)_{\zeta}\right)\right) d t d x \\
& \quad \leq \int_{Q} f T_{k}\left(v-\left(T_{h}(v)\right)_{\zeta}\right) d t d x+\int_{Q} G_{1} \cdot \nabla\left(T_{k}\left(v-\left(T_{h}(v)\right)_{\zeta}\right)\right) d t d x
\end{aligned}
$$


Since $\Theta_{k}$ is nonnegative, we have $\int_{\Omega} \Theta_{k}\left(v-\left(T_{h}(v)\right)_{\zeta}\right)(T) d x \geq 0$. Moreover, $v-\left(T_{h}(v)\right)_{\zeta}$ has the same sign as $T_{h}(v)-\left(T_{h}(v)\right)_{\zeta}$ : indeed, if $|v| \leq h$, then $v-$ $\left(T_{h}(v)\right)_{\zeta}=T_{h}(v)-\left(T_{h}(v)\right)_{\zeta}$ and, if $|v|>h$, since $\left|\left(T_{h}(v)\right)_{\zeta}\right| \leq h$ on $Q, v-\left(T_{h}(v)\right)_{\zeta}$ has the sign of $v$, which is also the sign of $T_{h}(v)-\left(T_{h}(v)\right)_{\zeta}=\operatorname{sgn}(v) h-\left(T_{h}(v)\right)_{\zeta}$. Therefore, since $\left(\left(T_{h}(v)\right)_{\zeta}\right)_{t} \in L^{1}(Q)$ has the same sign as $v-\left(T_{h}(v)\right)_{\zeta}$,

$$
\int_{0}^{T}\left\langle\left(\left(T_{h}(v)\right)_{\zeta}\right)_{t}, T_{k}\left(v-\left(T_{h}(v)\right)_{\zeta}\right)\right\rangle d t=\int_{Q}\left(\left(T_{h}(v)\right)_{\zeta}\right)_{t} T_{k}\left(v-\left(T_{h}(v)\right)_{\zeta}\right) d t d x \geq 0 .
$$

We deduce from this that

$$
\begin{aligned}
& \int_{Q} a(t, x, u, \nabla u) \cdot \nabla\left(T_{k}\left(v-\left(T_{h}(v)\right)_{\zeta}\right)\right) d t d x \\
& \leq \int_{\Omega} \Theta_{k}\left(u_{0}-z_{\zeta}\right) d x+\int_{Q} f T_{k}\left(v-\left(T_{h}(v)\right)_{\zeta}\right) d t d x \\
& \quad+\int_{Q} G_{1} \cdot \nabla\left(T_{k}\left(v-\left(T_{h}(v)\right)_{\zeta}\right)\right) d t d x
\end{aligned}
$$

As $\zeta \rightarrow \infty$, by the convergences (up to a subsequence) of $\left(T_{h}(v)\right)_{\zeta}$ to $T_{h}(v)$, we have $T_{k}\left(v-\left(T_{h}(v)\right)_{\zeta}\right)=T_{k}\left(T_{k+h}(v)-\left(T_{h}(v)\right)_{\zeta}\right) \rightarrow T_{k}\left(T_{k+h}(v)-T_{h}(v)\right)=$ $T_{k}\left(v-T_{h}(v)\right)$ in $L^{p}\left(0, T ; W_{0}^{1, p}(\Omega)\right)$ and a.e. on $Q$. Moreover, for all $\zeta>0$, we have $\left\{\nabla\left(T_{k}\left(v-\left(T_{h}(v)\right)_{\zeta}\right)\right) \neq 0\right\} \subset\left\{\left|v-\left(T_{h}(v)\right)_{\zeta}\right| \leq k\right\} \subset\{|v| \leq k+h\}$; thus, the integral sign in the left-hand side of $(6.2)$ can be taken on $\{|v| \leq k+h\}$ only (which does not depend on $\zeta$ ), and we have, on this set,

$$
\begin{aligned}
|a(t, x, u, \nabla u)| & \leq \beta\left(b+|u|^{\nu}+|\nabla u|^{p-1}\right) \mathbf{1}_{\{|v| \leq k+h\}} \\
& \leq \beta\left(b+|k+h+| g_{2}||^{\nu}+\left|\nabla\left(T_{k+h}(v)\right)+\nabla g_{2}\right|^{p-1}\right) \in L^{p^{\prime}}(Q) .
\end{aligned}
$$

Hence, we can pass to the limit $\zeta \rightarrow \infty$ in (6.2) to find

$$
\begin{aligned}
& \int_{Q} a(t, x, u, \nabla u) \cdot \nabla\left(T_{k}\left(v-T_{h}(v)\right)\right) d t d x \\
& \leq \int_{\Omega} \Theta_{k}\left(u_{0}-T_{h}\left(u_{0}\right)\right) d x+\int_{Q} f T_{k}\left(v-T_{h}(v)\right) d t d x \\
& \quad+\int_{Q} G_{1} \cdot \nabla\left(T_{k}\left(v-T_{h}(v)\right)\right) d t d x
\end{aligned}
$$

(we use the fact that $\left\{\nabla\left(T_{k}\left(v-T_{h}(v)\right)\right) \neq 0\right\}$ is also contained in $\{|v| \leq k+h\}$ ). But $\nabla\left(T_{k}\left(v-T_{h}(v)\right)\right)=\mathbf{1}_{\{h \leq|v| \leq h+k\}} \nabla v=\mathbf{1}_{\{h \leq|v| \leq h+k\}} \nabla u-\mathbf{1}_{\{h \leq|v| \leq h+k\}} \nabla g_{2}$, $\left|\Theta_{k}(s)\right| \leq k|s|$ and $T_{k}\left(v-T_{h}(v)\right)=0$ outside $\{|v| \geq h\}$, so that

$$
\begin{aligned}
& \int_{\{h \leq|v| \leq h+k\}}\left(\alpha|\nabla u|^{p}-\Lambda\right) d t d x \\
& \quad \leq \int_{Q} a(t, x, u, \nabla u) \cdot \nabla\left(T_{k}\left(v-T_{h}(v)\right)\right) d t d x
\end{aligned}
$$




$$
\begin{aligned}
& +\int_{\{h \leq|v| \leq h+k\}} a(t, x, u, \nabla u) \cdot \nabla g_{2} d t d x \\
\leq & k \int_{\Omega}\left|u_{0}-T_{h}\left(u_{0}\right)\right| d x+k \int_{\{|v| \geq h\}}|f| d t d x+\int_{\{h \leq|v| \leq h+k\}}\left|G_{1}\right||\nabla v| d t d x \\
& +\beta \int_{\{h \leq|v| \leq h+k\}}\left(b+|u|^{\nu}+|\nabla u|^{p-1}\right)\left|\nabla g_{2}\right| d t d x .
\end{aligned}
$$

Then, using Young's inequality and the fact that $|\nabla v| \leq|\nabla u|+\left|\nabla g_{2}\right|$, we find $C$ not depending on $h$ such that

$$
\begin{aligned}
& \alpha \int_{\{h \leq|v| \leq h+k\}}|\nabla u|^{p} d t d x \\
& \leq k \int_{\left\{\left|u_{0}\right| \geq h\right\}}\left|u_{0}\right| d x+k \int_{\{|v| \geq h\}}|f| d t d x \\
& \quad+C \int_{\{h \leq|v| \leq h+k\}}\left(\left|G_{1}\right|^{p^{\prime}}+\left|\nabla g_{2}\right|^{p}+b^{p^{\prime}}+|u|^{\nu p^{\prime}}\right) d t d x \\
& \quad+\frac{\alpha}{2} \int_{\{h \leq|v| \leq h+k\}}|\nabla u|^{p} d t d x+\int_{\{h \leq|v| \leq h+k\}} \Lambda d t d x .
\end{aligned}
$$

Thanks to Proposition 4.1, we see that the function $H=C\left(\left|G_{1}\right|^{p^{\prime}}+\left|\nabla g_{2}\right|^{p}+b^{p^{\prime}}+\right.$ $\left.|u|^{\nu p^{\prime}}\right)+\Lambda$ is in $L^{1}(Q)$, and, since meas $(\{|v| \geq h\}) \rightarrow 0$ as $h \rightarrow \infty$ ( $v$ is a.e. finite), the inequality

$$
\begin{aligned}
& \frac{\alpha}{2} \int_{\{h \leq|v| \leq h+k\}}|\nabla u|^{p} d t d x \\
& \quad \leq k \int_{\left\{\left|u_{0}\right| \geq h\right\}}\left|u_{0}\right| d x+k \int_{\{|v| \geq h\}}|f| d t d x+\int_{\{h \leq|v| \leq h+k\}} H d t d x
\end{aligned}
$$

implies (6.1).

\subsection{Conclusion}

We prove now that the entropy solution $u$ satisfies all the other properties of the renormalized solution.

To this end, let $\widetilde{a}(t, x, \xi)=a(t, x, u(t, x), \xi)$. By Proposition (4.1), $|u|^{\nu}$ is in $L^{p^{\prime}}(Q)$, so that $\widetilde{b}=b+|u|^{\nu} \in L^{p^{\prime}}(Q)$. The function $\widetilde{a}$ satisfies

$$
|\widetilde{a}(t, x, \xi)| \leq \beta\left(\widetilde{b}+|\xi|^{p-1}\right) \quad \text { for a.e. }(t, x) \in Q \text { and all } \xi \in \mathbf{R}^{N}
$$

and (2.1), (2.2), (2.4) (without the dependence in $s$ ). Hence, [6] gives $\left({ }^{2}\right)$ the

\footnotetext{
${ }^{2}$ In fact, in [6], there is no $\Lambda$ in $(2.2)$, but it is quite easy to see that the results of [6] still hold with $\Lambda$.
} 
existence of a renormalized solution $\widetilde{u}$ to

$$
\begin{cases}\widetilde{u}_{t}-\operatorname{div}(\widetilde{a}(t, x, \nabla \widetilde{u}))=\mu & \text { in } Q=] 0, T[\times \Omega, \\ \widetilde{u}=0 & \text { on }] 0, T[\times \partial \Omega, \\ \widetilde{u}(0)=u_{0} & \text { in } \Omega .\end{cases}
$$

We will now prove, using the same kind of tools as in the proof of uniqueness in [6], that $u=\widetilde{u}$. This will show that $u$ is a renormalized solution of (6.4), i.e. that it satisfies $(2.5),(2.6)$ (we have already proved these two properties in Proposition 4.1 and $(6.1)),(2.7)$ (since $\widetilde{a}(t, x, \nabla u)=a(t, x, u, \nabla u))$ and $(2.8)$.

Let $\widetilde{v}=\widetilde{u}-g_{2}$. Since $\widetilde{u}$ is a renormalized solution, we have $S_{n}(\widetilde{v}) \in E$ (see section 4). Using this test function in (2.11), we find

$$
\begin{aligned}
& \int_{\Omega} \Theta_{k}\left(v-S_{n}(\widetilde{v})\right)(T) d x-\int_{\Omega} \Theta_{k}\left(u_{0}-S_{n}\left(u_{0}\right)\right) d x \\
& \quad+\int_{0}^{T}\left\langle\left(S_{n}(\widetilde{v})\right)_{t}, T_{k}\left(v-S_{n}(\widetilde{v})\right)\right\rangle d t \\
& \quad+\int_{Q} \widetilde{a}(t, x, \nabla u) \cdot \nabla\left(T_{k}\left(v-S_{n}(\widetilde{v})\right)\right) d t d x \\
& \quad \leq \int_{Q} f T_{k}\left(v-S_{n}(\widetilde{v})\right) d t d x+\int_{\Omega} G_{1} \cdot \nabla\left(T_{k}\left(v-S_{n}(\widetilde{v})\right)\right) d t d x
\end{aligned}
$$

(recall that $\widetilde{a}(t, x, \nabla u)=a(t, x, u, \nabla u))$.

Since $S_{n}$ is bounded by $n+1$, we have $T_{k}\left(v-S_{n}(\widetilde{v})\right)=T_{k}\left(T_{k+n+1}(v)-\right.$ $\left.S_{n}(\widetilde{v})\right) \in L^{p}\left(0, T ; W_{0}^{1, p}(\Omega)\right) \cap L^{\infty}(Q)$; hence we can use this test function in the equation satisfied by $\widetilde{u}$ (renormalized solution to (6.4)) to obtain

$$
\begin{aligned}
& \int_{0}^{T}\left\langle\left(S_{n}(\widetilde{v})\right)_{t}, T_{k}\left(v-S_{n}(\widetilde{v})\right)\right\rangle d t \\
& =\int_{Q} f S_{n}^{\prime}(\widetilde{v}) T_{k}\left(v-S_{n}(\widetilde{v})\right) d t d x+\int_{Q} S_{n}^{\prime}(\widetilde{v}) G_{1} \cdot \nabla\left(T_{k}\left(v-S_{n}(\widetilde{v})\right)\right) d t d x \\
& \quad+\int_{Q} S_{n}^{\prime \prime}(\widetilde{v}) G_{1} \cdot \nabla \widetilde{v} T_{k}\left(v-S_{n}(\widetilde{v})\right) d t d x \\
& \quad-\int_{Q} S_{n}^{\prime \prime}(\widetilde{v}) \widetilde{a}(t, x, \nabla \widetilde{u}) \cdot \nabla \widetilde{v} T_{k}\left(v-S_{n}(\widetilde{v})\right) d t d x \\
& \quad-\int_{Q} S_{n}^{\prime}(\widetilde{v}) \widetilde{a}(t, x, \nabla \widetilde{u}) \cdot \nabla\left(T_{k}\left(v-S_{n}(\widetilde{v})\right)\right) d t d x
\end{aligned}
$$

We have $S_{n}^{\prime \prime}(s)=0$ if $|s| \notin[n, n+1]$ and $\left|S_{n}^{\prime \prime}\right| \leq 1$, so that, using (6.3),

$$
\begin{aligned}
\mid \int_{Q} S_{n}^{\prime \prime}(\widetilde{v}) G_{1} \cdot \nabla \widetilde{v} T_{k}\left(v-S_{n}(\widetilde{v})\right) d t d x \\
\quad-\int_{Q} S_{n}^{\prime \prime}(\widetilde{v}) \widetilde{a}(t, x, \nabla \widetilde{u}) \cdot \nabla \widetilde{v} T_{k}\left(v-S_{n}(\widetilde{v})\right) d t d x \mid
\end{aligned}
$$




$$
\begin{aligned}
\leq & C k \int_{\{n \leq|\widetilde{v}| \leq n+1\}}\left(\left|G_{1}\right|^{p^{\prime}}+\left|\nabla g_{2}\right|^{p}+|\nabla \widetilde{u}|^{p}\right) \\
& +C k \int_{\{n \leq|\widetilde{v}| \leq n+1\}}\left(\widetilde{b}^{p^{\prime}}+\left|\nabla g_{2}\right|^{p}+|\nabla \widetilde{u}|^{p}\right) d t d x \\
\leq & \omega_{1}(n)
\end{aligned}
$$

where $C$ does not depend on $n$ and $\omega_{1}(n) \rightarrow 0$ as $n \rightarrow \infty$ (property (2.6) of $\widetilde{u}$ ). Thus,

$$
\begin{aligned}
& \int_{0}^{T}\left\langle\left(S_{n}(\widetilde{v})\right)_{t}, T_{k}\left(v-S_{n}(\widetilde{v})\right)\right\rangle d t \\
& \geq-\omega_{1}(n)+\int_{Q} f S_{n}^{\prime}(\widetilde{v}) T_{k}\left(v-S_{n}(\widetilde{v})\right) d t d x \\
& \quad+\int_{Q} S_{n}^{\prime}(\widetilde{v}) G_{1} \cdot \nabla\left(T_{k}\left(v-S_{n}(\widetilde{v})\right)\right) d t d x \\
& \quad-\int_{Q} S_{n}^{\prime}(\widetilde{v}) \widetilde{a}(t, x, \nabla \widetilde{u}) \cdot \nabla\left(T_{k}\left(v-S_{n}(\widetilde{v})\right) d t d x .\right.
\end{aligned}
$$

Used in (6.5), this inequality gives, since $\Theta_{k}$ is nonnegative,

$$
\begin{aligned}
& \int_{Q}\left(\widetilde{a}(t, x, \nabla u)-S_{n}^{\prime}(\widetilde{v}) \widetilde{a}(t, x, \nabla \widetilde{u})\right) \cdot \nabla\left(T_{k}\left(v-S_{n}(\widetilde{v})\right)\right) d t d x \\
& \leq \int_{Q}\left(1-S_{n}^{\prime}(\widetilde{v})\right) f T_{k}\left(v-S_{n}(\widetilde{v})\right) d t d x \\
& \quad+\int_{\Omega}\left(1-S_{n}^{\prime}(\widetilde{v})\right) G_{1} \cdot \nabla\left(T_{k}\left(v-S_{n}(\widetilde{v})\right)\right) d t d x \\
& \quad+\int_{\Omega} \Theta_{k}\left(u_{0}-S_{n}\left(u_{0}\right)\right) d x+\omega_{1}(n) .
\end{aligned}
$$

Let us split the left-hand side of (6.6):

$$
\begin{aligned}
& \int_{Q}\left(\widetilde{a}(t, x, \nabla u)-S_{n}^{\prime}(\widetilde{v}) \widetilde{a}(t, x, \nabla \widetilde{u})\right) \cdot \nabla\left(T_{k}\left(v-S_{n}(\widetilde{v})\right)\right) d t d x \\
& \left.=\int_{\{|\widetilde{v}| \leq n\}}\left(\widetilde{a}(t, x, \nabla u)-S_{n}^{\prime}(\widetilde{v}) \widetilde{a}(t, x, \nabla \widetilde{u})\right)\right) \cdot \nabla\left(T_{k}\left(v-S_{n}(\widetilde{v})\right)\right) d t d x \\
& \quad+\int_{\{|\widetilde{v}|>n\}} \widetilde{a}(t, x, \nabla u) \cdot \nabla\left(T_{k}\left(v-S_{n}(\widetilde{v})\right)\right) d t d x \\
& \quad-\int_{\{|\widetilde{v}|>n\}} S_{n}^{\prime}(\widetilde{v}) \widetilde{a}(t, x, \nabla \widetilde{u}) \cdot \nabla\left(T_{k}\left(v-S_{n}(\widetilde{v})\right)\right) d t d x .
\end{aligned}
$$

On the set $\{|\widetilde{v}| \leq n\}$, we have $S_{n}(\widetilde{v})=\widetilde{v}, S_{n}^{\prime}(\widetilde{v})=1$ and $\nabla\left(T_{k}\left(v-S_{n}(\widetilde{v})\right)\right)=$ $\mathbf{1}_{\left\{\left|v-S_{n}(\widetilde{v})\right| \leq k\right\}}\left(\nabla v-S_{n}^{\prime}(\widetilde{v}) \nabla \widetilde{v}\right)=\mathbf{1}_{\{|v-\widetilde{v}| \leq k\}}(\nabla v-\nabla \widetilde{v})=\mathbf{1}_{\{|u-\widetilde{u}| \leq k\}}(\nabla u-\nabla \widetilde{u}) ;$ 
hence,

$$
\begin{aligned}
& \int_{\{|\widetilde{v}| \leq n\}}\left(\widetilde{a}(t, x, \nabla u)-S_{n}^{\prime}(\widetilde{v}) \widetilde{a}(t, x, \nabla \widetilde{u})\right) \cdot \nabla\left(T_{k}\left(v-S_{n}(\widetilde{v})\right)\right) d t d x \\
& =\int_{\{|\widetilde{v}| \leq n\}} \mathbf{1}_{\{|u-\widetilde{u}| \leq k\}}(\widetilde{a}(t, x, \nabla u)-\widetilde{a}(t, x, \nabla \widetilde{u})) \cdot(\nabla u-\nabla \widetilde{u}) d t d x .
\end{aligned}
$$

If $|\widetilde{v}| \geq n$, we have $n \leq\left|S_{n}(\widetilde{v})\right| \leq n+1$ (the second inequality is always true). Therefore, if $|\widetilde{v}| \geq n$ and $\left|v-S_{n}(\widetilde{v})\right| \leq k$, then $|v| \leq k+\left|S_{n}(\widetilde{v})\right| \leq k+n+1$ and $|v| \geq\left|S_{n}(\widetilde{v})\right|-k \geq n-k$. Since $S_{n}^{\prime}=0$ outside $[-n-1, n+1]$ and $\left|S_{n}^{\prime}\right| \leq 1$, we deduce that

$$
\begin{aligned}
& \left|\int_{\{|\widetilde{v}|>n\}} \widetilde{a}(t, x, \nabla u) \cdot \nabla\left(T_{k}\left(v-S_{n}(\widetilde{v})\right)\right) d t d x\right| \\
& =\left|\int_{\left\{|\widetilde{v}|>n,\left|v-S_{n}(\widetilde{v})\right| \leq k\right\}} \widetilde{a}(t, x, \nabla u) \cdot\left(\nabla v-S_{n}^{\prime}(\widetilde{v}) \nabla \widetilde{v}\right) d t d x\right| \\
& \leq\left|\int_{\left\{|\widetilde{v}|>n,\left|v-S_{n}(\widetilde{v})\right| \leq k\right\}} \widetilde{a}(t, x, \nabla u) \cdot \nabla v d t d x\right| \\
& \quad+\left|\int_{\left\{|\widetilde{v}|>n,\left|v-S_{n}(\widetilde{v})\right| \leq k\right\}} S_{n}^{\prime}(\widetilde{v}) \widetilde{a}(t, x, \nabla u) \cdot \nabla \widetilde{v} d t d x\right| \\
& \leq \quad \int_{\{n-k \leq|v| \leq n+k+1\}}\left(\widetilde{b}+|\nabla u|^{p-1}\right)|\nabla v| \\
& \quad+\beta \int_{\{n<|\widetilde{v}| \leq n+1, n-k \leq|v| \leq n+k+1\}}\left(\widetilde{b}+|\nabla u|^{p-1}\right)|\nabla \widetilde{v}| d t d x \\
& \leq \quad C \int_{\{n-k \leq|v| \leq n+k+1\}}\left(\widetilde{b}^{p^{\prime}}+|\nabla u|^{p}+\left|\nabla g_{2}\right|^{p}\right) d t d x+C \int_{\{n<|\widetilde{v}| \leq n+1\}}|\nabla \widetilde{u}|^{p} d t d x \\
& =\omega_{2}(n)
\end{aligned}
$$

with $C$ not depending on $n$ and $\omega_{2}(n) \rightarrow 0$ as $n \rightarrow \infty$ (we use (6.1) and the property of renormalized solution of $\widetilde{u})$. We can also bound, using the same properties of $S_{n}$ and $S_{n}^{\prime}$, the last term of (6.7):

$$
\begin{aligned}
& \left|\int_{\{|\widetilde{v}|>n\}} S_{n}^{\prime}(\widetilde{v}) \widetilde{a}(t, x, \nabla \widetilde{u}) \cdot \nabla\left(T_{k}\left(v-S_{n}(\widetilde{v})\right)\right) d t d x\right| \\
& \quad \leq \beta \int_{\left\{n<|\widetilde{v}| \leq n+1,\left|v-S_{n}(\widetilde{v})\right| \leq k\right\}}\left(\widetilde{b}+|\nabla \widetilde{u}|^{p-1}\right)(|\nabla v|+|\nabla \widetilde{v}|) d t d x \\
& \leq C \int_{\{n<|\widetilde{v}| \leq n+1, n-k \leq|v| \leq n+k+1\}}\left(\widetilde{b}^{p^{\prime}}+|\nabla \widetilde{u}|^{p}+\left|\nabla g_{2}\right|^{p}+|\nabla u|^{p}\right) d t d x \\
& =\omega_{3}(n)
\end{aligned}
$$


where, as before, $C$ does not depend on $n$ and $\omega_{3}(n) \rightarrow 0$ as $n \rightarrow \infty$. Injecting (6.8), (6.9) and (6.10) in (6.7), we find

$$
\begin{aligned}
& \int_{Q}\left(\widetilde{a}(t, x, \nabla u)-S_{n}^{\prime}(\widetilde{v}) \widetilde{a}(t, x, \nabla \widetilde{u})\right) \cdot \nabla\left(T_{k}\left(v-S_{n}(\widetilde{v})\right) d t d x\right. \\
& \quad \geq \int_{\{|\widetilde{v}| \leq n\}} \mathbf{1}_{\{|u-\widetilde{u}| \leq k\}}(\widetilde{a}(t, x, \nabla u)-\widetilde{a}(t, x, \nabla \widetilde{u})) \cdot(\nabla u-\nabla \widetilde{u}) d t d x-\omega_{4}(n)
\end{aligned}
$$

where $\omega_{4}(n) \rightarrow 0$ as $n \rightarrow \infty$. Using this inequality in (6.6), we deduce

$$
\begin{aligned}
& \int_{\{|\widetilde{v}| \leq n\}} \mathbf{1}_{\{|u-\widetilde{u}| \leq k\}}(\widetilde{a}(t, x, \nabla u)-\widetilde{a}(t, x, \nabla \widetilde{u})) \cdot(\nabla u-\nabla \widetilde{u}) d t d x \\
& \leq \omega_{5}(n)+k \int_{Q}|f|\left|1-S_{n}^{\prime}(\widetilde{v})\right| d t d x \\
& \quad+\int_{Q}\left|G_{1}\right|\left|1-S_{n}^{\prime}(\widetilde{v})\right|\left|\nabla\left(T_{k}\left(v-S_{n}(\widetilde{v})\right)\right)\right| d t d x \\
& \quad+k \int_{\Omega}\left|u_{0}-S_{n}\left(u_{0}\right)\right| d x
\end{aligned}
$$

with $\omega_{5}(n) \rightarrow 0$ as $n \rightarrow \infty$.

We have $S_{n}(s) \rightarrow s$ as $n \rightarrow \infty,\left|S_{n}(s)\right| \leq|s|, S_{n}^{\prime} \rightarrow 1$ and $\left|S_{n}^{\prime}\right| \leq 1$, so that the dominated convergence theorem gives

$$
\int_{\Omega}\left|u_{0}-S_{n}\left(u_{0}\right)\right| d x+\int_{Q}|f|\left|1-S_{n}^{\prime}(\widetilde{v})\right| d t d x \rightarrow 0 \quad \text { as } n \rightarrow \infty .
$$

Moreover, $S_{n}^{\prime}=1$ on $[-n, n], S_{n}^{\prime}=0$ outside $[-n-1, n+1]$, and $0 \leq S_{n}^{\prime} \leq 1$ so that

$$
\begin{aligned}
& \int_{Q}\left|G_{1}\right|\left|1-S_{n}^{\prime}(\widetilde{v})\right|\left|\nabla\left(T_{k}\left(v-S_{n}(\widetilde{v})\right)\right)\right| d t d x \\
& \leq \int_{\{|\widetilde{v}|>n\}}\left|G_{1}\right|\left|\nabla\left(T_{k}\left(v-S_{n}(\widetilde{v})\right)\right)\right| d t d x \\
& \leq \int_{\left\{|\widetilde{v}|>n,\left|v-S_{n}(\widetilde{v})\right| \leq k\right\}}\left(\left|G_{1}\right||\nabla v|+\left|G_{1}\right|\left|S_{n}^{\prime}(\widetilde{v})\right||\nabla \widetilde{v}|\right) d t d x \\
& \leq C \int_{\{n-k \leq|v| \leq n+k+1\}}\left(\left|G_{1}\right|^{p^{\prime}}+|\nabla u|^{p}+\left|\nabla g_{2}\right|^{p}\right) \\
& \quad+C \int_{\{n<|\widetilde{v}| \leq n+1\}}\left(\left|G_{1}\right|^{p^{\prime}}+|\nabla \widetilde{u}|^{p}+\left|\nabla g_{2}\right|^{p}\right) d t d x
\end{aligned}
$$

where $C$ does not depend on $n$ (we once again used $\left\{|\widetilde{v}|>n,\left|v-S_{n}(\widetilde{v})\right| \leq k\right\} \subset$ $\{n-k \leq|v| \leq n+k+1\})$. This last quantity going to 0 as $n \rightarrow \infty$, we can come 
back in (6.11) to see that

$$
\begin{aligned}
& \int_{Q} \mathbf{1}_{\{|\widetilde{v}| \leq n\}} \mathbf{1}_{\{|u-\widetilde{u}| \leq k\}}(\widetilde{a}(t, x, \nabla u)-\widetilde{a}(t, x, \nabla \widetilde{u})) \cdot(\nabla u-\nabla \widetilde{u}) d t d x \\
& \quad \leq \omega_{7}(n) \rightarrow 0 \quad \text { as } n \rightarrow \infty .
\end{aligned}
$$

Since $\mathbf{1}_{\{|u-\widetilde{u}| \leq k\}}(\widetilde{a}(t, x, \nabla u)-\widetilde{a}(t, x, \nabla \widetilde{u})) \cdot(\nabla u-\nabla \widetilde{u})$ is nonnegative, Fatou's lemma imply

$$
\int_{Q} \mathbf{1}_{\{|u-\widetilde{u}| \leq k\}}(\widetilde{a}(t, x, \nabla u)-\widetilde{a}(t, x, \nabla \widetilde{u})) \cdot(\nabla u-\nabla \widetilde{u}) d t d x \leq 0
$$

that is to say $\mathbf{1}_{\{|u-\widetilde{u}| \leq k\}}(\widetilde{a}(t, x, \nabla u)-\widetilde{a}(t, x, \nabla \widetilde{u})) \cdot(\nabla u-\nabla \widetilde{u})=0$ a.e. on $Q$ (thanks to (2.4)). This equality being valid for all $k \geq 0$, and $u-\widetilde{u}$ being a.e. finite, we deduce that $(\widetilde{a}(t, x, \nabla u)-\widetilde{a}(t, x, \nabla \widetilde{u})) \cdot(\nabla u-\nabla \widetilde{u})=0$ a.e. on $Q$, and thus that $\nabla u=\nabla \widetilde{u}$ a.e. on $Q$ (still using (2.4)). In particular, $\nabla v=\nabla \widetilde{v}$ a.e. on $Q$.

As in [6], we now conclude that $u=\widetilde{u}$. Considering $w_{n}=T_{1}\left(T_{n}(v)-T_{n}(\widetilde{v})\right) \in$ $L^{p}\left(0, T ; W_{0}^{1, p}(\Omega)\right)$, we have $\nabla w_{n}=\mathbf{1}_{\left\{\left|T_{n}(v)-T_{n}(\widetilde{v})\right| \leq 1\right\}}\left(\mathbf{1}_{\{|v| \leq n\}} \nabla v-\mathbf{1}_{\{|\widetilde{v}| \leq n\}} \nabla \widetilde{v}\right)$ so that

$$
\nabla w_{n}= \begin{cases}0 & \text { on }\{|v| \leq n,|\widetilde{v}| \leq n\} \cup\{|v|>n,|\widetilde{v}|>n\}, \\ \mathbf{1}_{\left\{\left|v-T_{n}(\widetilde{v})\right| \leq 1\right\}} \nabla v & \text { on }\{|v| \leq n,|\widetilde{v}|>n\} \\ -\mathbf{1}_{\left\{\left|\widetilde{v}-T_{n}(v)\right| \leq 1\right\}} \nabla \widetilde{v} & \text { on }\{|v|>n,|\widetilde{v}| \leq n\}\end{cases}
$$

But, if $|s|>n,|t| \leq n$ and $\left|t-T_{n}(s)\right| \leq 1$, then $n-1 \leq|t| \leq n$, which implies

$$
\begin{aligned}
\int_{Q}\left|\nabla w_{n}\right|^{p} d t d x \leq & \int_{\{n-1 \leq|v| \leq n\}}|\nabla v|^{p} d t d x \\
& +\int_{\{n-1 \leq|\widetilde{v}| \leq n\}}|\nabla \widetilde{v}|^{p} d t d x \\
\leq & C \int_{\{n-1 \leq|v| \leq n\}}\left(|\nabla u|^{p}+\left|\nabla g_{2}\right|^{p}\right) d t d x \\
& +C \int_{\{n-1 \leq|\widetilde{v}| \leq n\}}\left(|\nabla \widetilde{u}|^{p}+\left|\nabla g_{2}\right|^{p}\right) d t d x .
\end{aligned}
$$

By (6.1) and Property (2.6) of renormalized solutions, we see that $w_{n} \rightarrow 0$ in $L^{p}\left(0, T ; W_{0}^{1, p}(\Omega)\right)$, and thus in $\mathcal{D}^{\prime}(Q)$. Since $w_{n} \rightarrow T_{1}(v-\widetilde{v})$ a.e. and remains bounded by 1 , we also have $w_{n} \rightarrow T_{1}(v-\widetilde{v})$ in $\mathcal{D}^{\prime}(Q)$. Hence, $T_{1}(v-\widetilde{v})=0$, i.e. $v=\widetilde{v}$ on $Q$. This concludes the proof that $u=\widetilde{u}$ and that $u$ is a renormalized solution of (1.1).

\section{Appendix}

We prove here an integration by parts formula. A similar result exists in [8], but in the case where the derivative is not expressed as the sum of two functions in 
different spaces; the proof in this latter case is not completely straightforward (it demands an unusual regularization theorem of [5]), so we include it for the sake of completeness.

Lemma 7.1 Let $f: \mathbf{R} \rightarrow \mathbf{R}$ be a continuous piecewise $C^{1}$ function such that $f(0)=0$ and $f^{\prime}$ is zero away from a compact set of $\mathbf{R}$; let us denote $F(s)=$ $\int_{0}^{s} f(r) d r$. If $u \in L^{p}\left(0, T ; W_{0}^{1, p}(\Omega)\right)$ is such that $u_{t} \in L^{p^{\prime}}\left(0, T ; W^{-1, p^{\prime}}(\Omega)\right)+L^{1}(Q)$ and if $\psi \in C^{\infty}(\bar{Q})$, then we have

$$
\int_{0}^{T}\left\langle u_{t}, f(u) \psi\right\rangle d t=\int_{\Omega} F(u(T)) \psi(T) d x-\int_{\Omega} F(u(0)) \psi(0) d x-\int_{Q} \psi_{t} F(u) d t d x
$$

(we have chosen the continuous representative of $u$ ).

Proof. One has $F(u) \in C\left([0, T] ; L^{1}(\Omega)\right)$, since $F^{\prime}$ is bounded and $u \in C([0, T]$; $\left.L^{1}(\Omega)\right)$. Moreover $\psi f(u) \in L^{p}\left(0, T ; W_{0}^{1, p}(\Omega)\right) \cap L^{\infty}(Q)$ since $f$ is bounded and piecewise $C^{1}$. Hence each term makes sense.

We denote' the derivative with respect to $t$ for sequences of functions (in order to avoid double indexing). Let $v^{1} \in L^{p^{\prime}}\left(0, T ; W^{-1, p^{\prime}}(\Omega)\right)$ and $v^{2} \in L^{1}(Q)$ such as $u_{t}=v^{1}+v^{2}$. Thanks to Corollary 2.3.2 of [5, p. 50], there exists $u_{n} \in$ $C^{\infty}\left([0, T] ; W_{0}^{1, p}(\Omega)\right), \quad v_{n}^{1} \in C^{\infty}\left([0, T] ; W^{-1, p^{\prime}}(\Omega)\right)$ and $v_{n}^{2} \in C^{\infty}\left([0, T] ; L^{1}(\Omega)\right)$ such that $u_{n}^{\prime}=v_{n}^{1}+v_{n}^{2}$ and $u_{n} \rightarrow u$ in $L^{p}\left(0, T ; W_{0}^{1, p}(\Omega)\right), v_{n}^{1} \rightarrow v^{1}$ in $L^{p^{\prime}}(0, T$; $\left.W^{-1, p^{\prime}}(\Omega)\right)$ and $v_{n}^{2} \rightarrow v^{2}$ in $L^{1}\left(0, T ; L^{1}(\Omega)\right)$.

Since $F$ is $C^{1}, F^{\prime}=f$ is bounded and $u_{n} \in W^{1,1}\left(0, T ; L^{p}(\Omega)\right)$, one has $\psi F\left(u_{n}\right) \in W^{1,1}\left(0, T ; L^{p}(\Omega)\right)$; hence, in $L^{p}(\Omega)$,

$$
\begin{aligned}
\psi(T) F\left(u_{n}(T)\right)-\psi(0) F\left(u_{n}(0)\right) & =\int_{0}^{T}\left(\psi F\left(u_{n}\right)\right)^{\prime} d t \\
& =\int_{0}^{T} \psi_{t} F\left(u_{n}\right) d t+\int_{0}^{T} \psi f\left(u_{n}\right) u_{n}^{\prime} d t
\end{aligned}
$$

and

$$
\begin{aligned}
\int_{\Omega} \psi(T) F\left(u_{n}(T)\right) d x-\int_{\Omega} \psi(0) F\left(u_{n}(0)\right) d x \\
=\int_{Q} \psi_{t} F\left(u_{n}\right) d t d x+\int_{Q} \psi f\left(u_{n}\right) u_{n}^{\prime} d t d x
\end{aligned}
$$

We have $u_{n}^{\prime}(t)=v_{n}^{1}(t)+v_{n}^{2}(t)$ and, since $\psi(t) f\left(u_{n}(t)\right) \in W_{0}^{1, p}(\Omega) \cap L^{\infty}(\Omega)$ (because $f(0)=0$ and $f^{\prime}$ is bounded), we deduce that

$$
\begin{aligned}
\int_{\Omega} \psi(t) f\left(u_{n}(t)\right) u_{n}^{\prime}(t) d x= & \left\langle v_{n}^{1}(t), \psi(t) f\left(u_{n}(t)\right)\right\rangle_{W^{-1, p^{\prime}}(\Omega), W_{0}^{1, p}(\Omega)} \\
& +\int_{\Omega} v_{n}^{2}(t) \psi(t) f\left(u_{n}(t)\right) d x
\end{aligned}
$$


Therefore,

$$
\begin{aligned}
\int_{\Omega} \psi & (T) F\left(u_{n}(T)\right) d x-\int_{\Omega} \psi(0) F\left(u_{n}(0)\right) d x \\
= & \int_{Q} \psi_{t} F\left(u_{n}\right) d t d x+\int_{0}^{T}\left\langle v_{n}^{1}, \psi f\left(u_{n}\right)\right\rangle_{W^{-1, p^{\prime}}(\Omega), W_{0}^{1, p}(\Omega)} d t \\
& \quad+\int_{Q} v_{n}^{2} \psi f\left(u_{n}\right) d t d x .
\end{aligned}
$$

According to the choice of $u_{n}$, we have $u_{n} \rightarrow u$ in $L^{p}\left(0, T ; W_{0}^{1, p}(\Omega)\right)$ and $u_{n}^{\prime} \rightarrow u_{t}$ in $L^{p^{\prime}}\left(0, T ; W^{-1, p^{\prime}}(\Omega)\right)+L^{1}(Q)$; thus, $u_{n} \rightarrow u$ in $C\left([0, T] ; L^{1}(\Omega)\right)$ (see [9]). We also have $u_{n} \rightarrow u$ in $L^{p}(Q)$ and therefore, up to a subsequence, $u_{n} \rightarrow u$ almost everywhere in $Q$.

Since $f=F^{\prime}$ is bounded, $F\left(u_{n}\right) \rightarrow F(u)$ in $C\left([0, T] ; L^{1}(\Omega)\right) \hookrightarrow L^{1}(Q)$, and $F\left(u_{n}(T)\right) \rightarrow F(u(T))$ and $F\left(u_{n}(0)\right) \rightarrow F(u(0))$ in $L^{1}(\Omega)$; hence

$$
\begin{aligned}
\lim _{n \rightarrow \infty} & \int_{\Omega} \psi(T) F\left(u_{n}(T)\right) d x-\int_{\Omega} \psi(0) F\left(u_{n}(0)\right) d x \\
& =\int_{\Omega} \psi(T) F(u(T)) d x-\int_{\Omega} \psi(0) F(u(0)) d x
\end{aligned}
$$

and

$$
\lim _{n \rightarrow \infty} \int_{Q} \psi_{t} F\left(u_{n}\right) d t d x=\int_{Q} \psi_{t} F(u) d t d x .
$$

Since $f$ is continuous piecewise $C^{1}$ with a bounded derivative and $u_{n} \rightarrow u$ in $L^{p}\left(0, T ; W_{0}^{1, p}(\Omega)\right)$, one has $\psi f\left(u_{n}\right) \rightarrow \psi f(u)$ in $L^{p}\left(0, T ; W_{0}^{1, p}(\Omega)\right)$. Using the fact that $v_{n}^{1} \rightarrow v^{1}$ in $L^{p^{\prime}}\left(0, T ; W^{-1, p^{\prime}}(\Omega)\right)$, we obtain

$$
\begin{aligned}
\lim _{n \rightarrow \infty} & \int_{0}^{T}\left\langle v_{n}^{1}, \psi f\left(u_{n}\right)\right\rangle_{W^{-1, p^{\prime}}(\Omega), W_{0}^{1, p}(\Omega)} d t \\
& =\int_{0}^{T}\left\langle v^{1}, \psi f(u)\right\rangle_{W^{-1, p^{\prime}}(\Omega), W_{0}^{1, p}(\Omega)} d t .
\end{aligned}
$$

Since $f$ is bounded, $\psi f\left(u_{n}\right)$ tends to $\psi f(u)$ almost everywhere on $Q$ and remains bounded; hence, the convergence holds in $L^{\infty}(Q)$ weak-*. Since $v_{n}^{2} \rightarrow v^{2}$ in $L^{1}(Q)$, we get

$$
\lim _{n \rightarrow \infty} \int_{Q} v_{n}^{2} \psi f\left(u_{n}\right) d t d x=\int_{Q} v^{2} \psi f(u) d t d x
$$

We can now pass to the limit in (7.1), and the proof is concluded. 


\section{References}

[1] P. BENILAN, L. BOCCARDO, T. GALLOUËT, R. GARIEPY, M. PIERRE and J. L. VAZQUEZ, An $L^{1}$-Theory of Existence and Uniqueness of Solutions of Nonlinear Elliptic Equations, Ann. Scuola Norm. Sup. Pisa Sci. Fis. Mat., IV 22(2) (1995).

[2] D. BLANCHARD and F. MURAT, Renormalized solutions of nonlinear parabolic problems with $L^{1}$ data: existence and uniqueness, Proc. of the Royal Soc. of Edinburgh Section A 127 (1997), 1137-1152.

[3] L. BOCCARDO, A. DALL'AGLIO, T. GALLOUËT and L. ORSINA, Nonlinear parabolic equations with measure data, Journ. of Functional Anal. 147 (1997), 237-258.

[4] L. BOCCARDO and T. GALLOUËT, Nonlinear elliptic and parabolic equations involving measure data, J. Funct. Anal. 87 (1989), 241-273.

[5] J. DRONIOU, Intégration et Espaces de Sobolev à valeurs vectorielles, available at http://www-gm3.univ-mrs.fr/polys/

[6] J. DRONIOU, A. PORRETTA and A. PRIGNET, Parabolic Capacity and soft measures for nonlinear equations, Potential Analysis 19(2) (2003), $99-161$.

[7] R. LANDES, On the existence of weak solutions for quasilinear parabolic boundary value problems, Proc. Royal Soc. Edinburgh Sect. A 89 (1981), $217-237$.

[8] G. GAGNEUX and M. MADAUNE-TORT, Analyse mathématique de modèles non linéaires de l'ingéniérie pétrolière, Mathématiques et Applications, 22 Springer, 1996.

[9] A. PORRETTA, Existence results for nonlinear parabolic equations via strong convergence of truncations, Ann. Mat. Pura ed Appl. (IV) 177 (1999), $143-172$.

[10] A. PRIGNET, Existence and uniqueness of "entropy" solutions of parabolic problems with $L^{1}$ data, Nonlin. Anal. TMA 28 (1997), 1943-1954.

Received 16 June 2005; accepted 16 October 2005

To access this journal online:

http://www.birkhauser.ch 